\title{
The Quantum Corrected Mode Function and Power Spectrum for a Scalar Field during Inflation
}

\author{
V. K. Onemli* \\ *Department of Physics, Istanbul Technical University, Maslak, Istanbul 34469, Turkey
}

We compute the one- and two-loop corrected mode function of a massless minimally coupled scalar endowed with a quartic self-interaction in the locally de Sitter background of an inflating universe for a state which is released in Bunch-Davies vacuum at time $t=0$. We then employ it to correct the scalar's tree-order scale invariant power spectrum $\Delta_{\varphi}^{2}$. The corrections are secular, and have scale dependent part that can be expanded in even powers of $k /(H a)$, where $k$ is the comoving wave number, $H$ is the expansion rate and $a$ is the cosmic scale factor. At one-loop, the scale invariant shift in the power spectrum grows as $(H t)^{2}$ in leading order. The $k$-dependent shifts, however, are constants for each mode, in the late time limit. At two-loop order, on the other hand, the scale invariant shift grows as $(H t)^{4}$ whereas the $k$-dependent shifts grow as $(H t)^{2}$, in leading order. We finally calculate the scalar's spectral index $n_{\varphi}$ and the running of the spectral index $\alpha_{\varphi}$. They imply that the spectrum is slightly red-tilted; hence, the amplitudes of fluctuations grow slightly towards the larger scales.

PACS numbers: 98.80.Cq, 04.62.+v

\section{INTRODUCTION}

Inflationary expansion enhances quantum effects [1]. The enhancement can be strong enough for quantum fields that are classically conformally non-invariant and effectively massless, to be realized even on cosmic scales. Massless minimally coupled (MMC) scalars and

\footnotetext{
*Electronic address: onemli@itu.edu.tr
} 
gravitons are the two examples which possess these attributes. The observed scalar perturbations [2] and potentially observable tensor perturbations [3] are, indeed, the amplified imprints of tiny quantum fluctuations of inflatons (inflationary scalars) and gravitons on the cosmic microwave anisotropy, respectively.

Thus, to study further possible quantum effects on cosmological scales, we considered, in Refs. [4-8], the MMC scalar field with a quartic self-interaction $\lambda \varphi^{4}(x)$ in the locally de Sitter spacetime. It is the cosmological constant $\Lambda$ that drives inflation in the model. The constant expansion rate $H=\sqrt{\Lambda /(D-1)}$ in $D$-spacetime dimensions. The scalar $\varphi$ is a spectator to the $\Lambda$-driven de Sitter inflation. We revealed several intriguing quantum aspects of the model. The fully renormalized vacuum expectation value of the energy density $\rho_{\text {ren }}$ and pressure $p_{\text {ren }}$ were computed [4-6] using dimensional regularization at one and two-loop orders. Although the classical covariant stress-energy conservation law $\dot{\rho}=-(D-1) H(\rho+p)$ is obeyed by $\rho_{\text {ren }}$ and $p_{\text {ren }}$, the classical weak energy condition (WEC) $\rho+p \geq 0$ is violated on cosmological scales - on average, not just in fluctuations. This quantum anomaly is a two-loop effect. The parameter $w \equiv p_{\text {ren }} / \rho_{\text {ren }}<-1$ at that order, thus, a temporary phase of super-acceleration is induced as a quantum effect. Physically, the effect can be described as follows. Inflationary particle production implies the growth of the scalar field strength. The scalar undergoes a random walk, so that, its position in the quartic potential rises, on average. Hence, the vacuum energy density increases: $\dot{\rho}_{\text {ren }}>0$. Combined with this fact, the stress-energy conservation implies the violation of the WEC: $\rho_{\text {ren }}+p_{\text {ren }}<0$. This process, however, is self-limiting. Because the field develops [7] a positive self-mass squared as it grows, the particle production is cut off. Moreover, as the scalar rises up its potential the classical restoring force pushes it back down. The field cannot continue rolling up its position and comes to a halt. Hence, the effect in the system is self-terminated.

The stability of the system was studied in Ref. [8]. We putatively solved the linearized Schwinger-Keldish effective scalar field equation in the late time limit $t \gg 0$, in leading logarithm order, and obtained the mode function as

$$
\Phi(x, \vec{k}) \sim u(\infty, k) e^{i \vec{k} \cdot \vec{x}}\left\{1-\frac{\lambda}{2^{4} 3 \pi^{2}}\left[\ln ^{2}(a)-\frac{2 \ln (a)}{3}\right]+\frac{\lambda^{2}}{2^{8} 3^{3} \pi^{4}}\left[\frac{11 \ln ^{4}(a)}{2}-\ln ^{3}(a)\right]+\mathcal{O}\left(\lambda^{3}\right)\right\} .
$$

Here $\vec{k}$ is the comoving wavevector, $k$ is the comoving wave number and $a=e^{H t}$ is the scale factor of the spacetime. The amplitude $u(\infty, k)=H / \sqrt{2 k^{3}}$ is the classical Bunch-Davies mode function $u(t, k)$ in the late time limit $t \gg 0$. Equation (1) implies that the perturbation 
breaks down when $\ln (a) \sim 1 / \sqrt{\lambda}$. For a coupling constant $\lambda \ll 1$, one can have a long time evolution during which the perturbation theory is still valid. As time evolves, the amplitude is reduced. The linear perturbations do not grow in this model. So, the model is stable.

Solution (1) for $\Phi(x, \vec{k})$ is correct in leading logarithm order. However, due to the restrictions [8] imposed on the solution, it neglects the sub-leading terms and completely misses the wave number $k$-dependent shifts that are essential in revealing the scale dependence of the scalar's power spectrum. In de Sitter background, the one-loop corrections to the mode functions in several different models have been calculated, in leading logarithm order, under similar restrictions. The one-loop corrections to the fermion and scalar mode functions for a MMC scalar Yukawa coupled to a massless fermion were calculated in Refs. [9] and [10], respectively. The one-loop corrections to the photon and scalar mode functions in MMC scalar quantum electrodynamics were calculated in Refs. [12] and [13], respectively. The one-loop correction to the fermion mode function for a massless fermion coupled to gravity was calculated in Ref. [14]. The one-loop correction to the scalar mode function for a MMC scalar coupled to gravity was calculated in Ref. [15]. As in Eq. (1) these solutions were putative and neglected sub-leading terms. Except for the one in Ref. [15], they did not include the $k$-dependent shifts.

A purpose of the present paper is to calculate the full one- and the two-loop corrections to the classical Bunch-Davies scalar mode function in our model. This is achieved by exactly solving the linearized Schwinger-Keldish effective scalar field equation, including the fully nonlocal and derivative corrections, by applying the Green's function technique. In both loop orders, as we show, the quantum corrections induce $k$-dependent shifts to the scalar mode function. We also analyze how these shifts modify the scalar's scale invariant tree-order power spectrum, its spectral index and the running of the spectral index in this paper.

The outline is as follows. In Sec. II we specify the background geometry, present the model, and briefly review the computation of the tree-order (Bunch-Davies) scalar mode function. We obtain the linearized Schwinger-Keldish effective scalar field equation at oneand two-loop orders in Sec. III and solve it exactly in Sec. IV. We calculate the scalar's quantum corrected power spectrum, its spectral index and the running of the spectral index at one- and two-loop orders in Sec. V. Implications of our results are discussed in Sec. VI. The Appendices comprise various key steps of the computations in the paper. 


\section{THE MODEL}

In our model, the background metric $g_{\mu \nu}$ describes open conformal coordinate patch of de Sitter spacetime. The invariant line element can be expressed in conformal and comoving coordinates, respectively, as

$$
d s^{2}=g_{\mu \nu} d x^{\mu} d x^{\nu}=a^{2}(\eta)\left[-d \eta^{2}+d \vec{x} \cdot d \vec{x}\right]=-d t^{2}+e^{2 H t} d \vec{x} \cdot d \vec{x}
$$

where the conformal factor

$$
a(\eta)=-\frac{1}{H \eta}=e^{H t}
$$

To exploit dimensional regularization we work in $D$ spacetime dimensions, hence the indices $\mu, \nu=0,1,2, \ldots,(D-1)$. In our notation: $x^{\mu}=\left(x^{0}, \vec{x}\right), x^{0} \equiv \eta$, and $\partial_{\mu}=\left(\partial_{0}, \vec{\nabla}\right)$.

The state is released in Bunch-Davies vacuum at comoving time $t=0$, corresponding to conformal time $\eta=\eta_{i} \equiv-H^{-1}$. Infinite future $t \rightarrow \infty$ corresponds to $\eta \rightarrow 0^{-}$. The conformal factor is normalized to $a=1$ when the state is released, hence $a>1$ throughout the evolution.

We take the Lagrangian density in our model, in terms of unrenormalized scalar field $\phi$, bare mass $m_{0}$ and bare coupling constant $\lambda_{0}$, as

$$
\mathcal{L}=-\frac{1}{2} \sqrt{-g} g^{\mu \nu} \partial_{\mu} \phi \partial_{\nu} \phi-\frac{m_{0}^{2}}{2} \sqrt{-g} \phi^{2}-\frac{\lambda_{0}}{4 !} \sqrt{-g} \phi^{4}
$$

Here, $g$ is the determinant of the metric $g_{\mu \nu}$. Introducing the renormalized scalar field

$$
\varphi(x) \equiv \frac{\phi(x)}{\sqrt{Z}},
$$

converts the Lagrangian density $\mathcal{L}$ to the form

$$
\mathcal{L}=-\frac{Z}{2} \sqrt{-g} g^{\mu \nu} \partial_{\mu} \varphi \partial_{\nu} \varphi-\frac{Z m_{0}^{2}}{2} \sqrt{-g} \varphi^{2}-\frac{Z^{2} \lambda_{0}}{4 !} \sqrt{-g} \varphi^{4}
$$

Renormalization is achieved by decomposing the bare parameters into renormalized and counter parameters as

$$
Z \equiv 1+\delta Z, \quad Z m_{0}^{2} \equiv 0+\delta m^{2}, \quad Z^{2} \lambda_{0} \equiv \lambda+\delta \lambda
$$

where we enforce the renormalized mass to be zero, initially. In time, however, quantum processes generate self-mass [7]. So, our scalar field $\varphi(x)$ is classically massless. Thus, the renormalized Lagrangian density is

$$
\mathcal{L}=-\frac{(1+\delta Z)}{2} \sqrt{-g} g^{\mu \nu} \partial_{\mu} \varphi \partial_{\nu} \varphi-\frac{\delta m^{2}}{2} \sqrt{-g} \varphi^{2}-\frac{(\lambda+\delta \lambda)}{4 !} \sqrt{-g} \varphi^{4}
$$


The field strength $(\delta Z)$, mass $\left(\delta m^{2}\right)$, and coupling constant $(\delta \lambda)$ counterterms remove divergences at one- and two-loop orders. They are given as functions of dimensional regularization parameter $\epsilon \equiv 4-D$ in Ref. [7]. Varying the Lagrangian with density (8) yields the scalar field equation

$$
\partial_{\mu}\left(\sqrt{-g} g^{\mu \nu} \partial_{\nu} \varphi\right)=\frac{\sqrt{-g}}{1+\delta Z}\left[\delta m^{2} \varphi+\frac{(\lambda+\delta \lambda)}{6} \varphi^{3}\right] .
$$

Its solution can be given as

$$
\varphi(x)=\varphi_{0}(x)+\frac{1}{1+\delta Z} \int_{\eta_{i}}^{0} d \eta^{\prime} \sqrt{-g\left(\eta^{\prime}\right)} \int d^{D-1} x^{\prime} G\left(x ; x^{\prime}\right)\left[\delta m^{2} \varphi\left(x^{\prime}\right)+\frac{(\lambda+\delta \lambda)}{6} \varphi^{3}\left(x^{\prime}\right)\right],
$$

where $\varphi_{0}(x)$ is the solution for the homogeneous equation

$$
\partial_{\mu}\left(\sqrt{-g} g^{\mu \nu} \partial_{\nu} \varphi_{0}(x)\right)=0,
$$

and the Green's function $G\left(x ; x^{\prime}\right)$ is any solution of the equation

$$
\partial_{\mu}\left(\sqrt{-g} g^{\mu \nu} \partial_{\nu} G\left(x ; x^{\prime}\right)\right)=\delta^{D}\left(x-x^{\prime}\right),
$$

which obeys retarded boundary conditions. It is the free scalar field $\varphi_{0}(x)$ what mainly concerns us, in this paper. How can we write the most general $\varphi_{0}(x)$ ? In conformal coordinates Eq. (11) becomes

$$
\varphi_{0}^{\prime \prime}(\eta, \vec{x})+(D-2) \frac{a^{\prime}}{a} \varphi_{0}^{\prime}(\eta, \vec{x})-\nabla^{2} \varphi_{0}(\eta, \vec{x})=0,
$$

where a prime denotes derivative with respect to the conformal time $\eta$. To find the solution for Eq. (13) we Fourier transform it

$$
\widetilde{\varphi}_{0}^{\prime \prime}(\eta, k)+(D-2) \frac{a^{\prime}}{a} \widetilde{\varphi}_{0}^{\prime}(\eta, k)+k^{2} \widetilde{\varphi}_{0}(\eta, k)=0,
$$

where

$$
\widetilde{\varphi}_{0}(\eta, k)=\int d^{D-1} x e^{-i \vec{k} \cdot \vec{x}} \varphi_{0}(\eta, \vec{x}),
$$

and look for a function $u(\eta, k)$ which satisfies Eq. (14), to wit,

$$
u^{\prime \prime}(\eta, k)+(D-2) \frac{a^{\prime}}{a} u^{\prime}(\eta, k)+k^{2} u(\eta, k)=0 .
$$

Making the transformation

$$
u(\eta, k) \equiv a^{-\frac{D-1}{2}} v(\eta, k),
$$

and using Eq. (3) brings differential equation (16) to the form

$$
v^{\prime \prime}(\eta, k)+\frac{v^{\prime}(\eta, k)}{\eta}+\left[k^{2}-\frac{\nu^{2}}{\eta^{2}}\right] v(\eta, k)=0,
$$


where $\nu \equiv \frac{D-1}{2}$. Making the change of variable $\eta=x / k$ reduces Eq. (18) to the Bessel's equation of order $\nu$

$$
\frac{d^{2} v(x)}{d x^{2}}+\frac{1}{x} \frac{d v(x)}{d x}+\left[1-\frac{\nu^{2}}{x^{2}}\right] v(x)=0,
$$

whose solution is given in terms of the first and second kind Hankel functions as

$$
v(\eta, k)=\mathcal{A H}_{\nu}^{(1)}(k \eta)+\mathcal{B H}_{\nu}^{(2)}(k \eta)
$$

The coefficients $\mathcal{A}$ and $\mathcal{B}$ are arbitrary constants. Therefore, the solution $u(\eta, k)$ of Eq. (16) can be given as

$$
u(\eta, k)=\mathcal{A} a^{-\frac{D-1}{2}} \mathcal{H}_{\frac{D-1}{2}}^{(1)}(k \eta)+\mathcal{B} a^{-\frac{D-1}{2}} \mathcal{H}_{\frac{D-1}{2}}^{(2)}(k \eta)
$$

The Bunch-Davies mode function is associated with the choice of $\mathcal{B}=0$.

Now, notice the analogy between the kinetic term of our Lagrangian

$$
L=\frac{a^{D-2}(\eta)}{2} \int \frac{d^{D-1} k}{(2 \pi)^{D-1}}\left[\left|\widetilde{\varphi}^{\prime}(\eta, k)\right|^{2}-k^{2}|\widetilde{\varphi}(\eta, k)|^{2}\right]
$$

and the Lagrangian of a simple harmonic oscillator

$$
L_{S H O}=\frac{m}{2} \int d t\left[\dot{q}^{2}(t)-\omega^{2} q^{2}(t)\right] .
$$

The correspondences between $\tilde{\varphi}(\eta, k) \leftrightarrow q(t), k \leftrightarrow \omega$ and $a^{D-2} \leftrightarrow m$ for each mode $k$, imply that $\widetilde{\varphi}(\eta, k)$ behaves like a simple harmonic oscillator. The well-known solution of the equation of motion for $q(t)$ in terms of the initial values $q_{I} \equiv q\left(t_{I}\right)$ and $\dot{q}_{I} \equiv \dot{q}\left(t_{I}\right)$ is

$$
q(t)=q_{I} \cos (\omega t)+\frac{\dot{q}_{I}}{\omega} \sin (\omega t)=\frac{e^{-i \omega t}}{2}\left[q_{I}+\frac{i \dot{q}_{I}}{\omega}\right]+\frac{e^{i \omega t}}{2}\left[q_{I}-\frac{i \dot{q}_{I}}{\omega}\right]
$$

The commutator of the operators $\left[q_{I}, \dot{q}_{I}\right]=i / m$. The annihilation and creation operators are respectively defined as the operators next to $e^{-i \omega t}$ and $e^{i \omega t}$,

$$
a(\omega)=\sqrt{\frac{m \omega}{2}}\left[q_{I}+\frac{i \dot{q}_{I}}{\omega}\right], \quad a^{\dagger}(\omega)=\sqrt{\frac{m \omega}{2}}\left[q_{I}-\frac{i \dot{q}_{I}}{\omega}\right] .
$$

The normalization factors are chosen to have $\left[a, a^{\dagger}\right]=1$. Thus,

$$
q(t)=\frac{e^{-i \omega t}}{\sqrt{2 m \omega}} a(\omega)+\frac{e^{i \omega t}}{\sqrt{2 m \omega}} a^{\dagger}(\omega),
$$

is the solution of the equation of motion $\ddot{q}(t)+\omega^{2} q(t)=0$ for each $\omega$.

Similarly, by analogy, the solution of equation of motion for the $\widetilde{\varphi}(\eta, k)$, for each mode $k$, can be written as

$$
\widetilde{\varphi}(\eta, k)=u(\eta, k) \alpha(\vec{k})+u^{*}(\eta, k) \alpha^{\dagger}(-\vec{k}) .
$$


Here, the mode function $u(\eta, k)$ is analogous to $\frac{e^{-i \omega t}}{\sqrt{2 m \omega}}$, in Eq. (26), that solves the equation of motion $\ddot{q}(t)+\omega^{2} q(t)=0$. Operators $\alpha(\vec{k}), \alpha^{\dagger}(\vec{k})$ are the annihilation and creation operators of the scalar and can be expressed in terms of initial values $a\left(\eta_{I}\right), u^{*}\left(\eta_{I}, k\right), u^{\prime *}\left(\eta_{I}, k\right), \widetilde{\varphi}\left(\eta_{I}, \vec{k}\right)$, and $\widetilde{\varphi}^{\prime}\left(\eta_{I}, \vec{k}\right)$, analogous to $a$ and $a^{\dagger}$. To see this, notice that the canonical commutation relations

$$
\begin{aligned}
{\left[\widetilde{\varphi}(\eta, \vec{k}), \Pi\left(\eta, \vec{k}^{\prime}\right)\right] } & =\left[\widetilde{\varphi}(\eta, \vec{k}), a^{D-2}(\eta) \widetilde{\varphi}^{\prime}\left(\eta, \vec{k}^{\prime}\right)\right]=i(2 \pi)^{D-1} \delta^{D-1}\left(\vec{k}-\vec{k}^{\prime}\right) \\
{\left[\alpha(\vec{k}), \alpha^{\dagger}\left(\vec{k}^{\prime}\right)\right] } & =(2 \pi)^{D-1} \delta^{D-1}\left(\vec{k}-\vec{k}^{\prime}\right)
\end{aligned}
$$

with

$$
\widetilde{\varphi}^{\prime}(\eta, k)=u^{\prime}(\eta, k) \alpha(\vec{k})+u^{\prime *}(\eta, k) \alpha^{\dagger}(-\vec{k}),
$$

fixes the Wronskian of differential equation (14) as

$$
W(\eta, k)=u(\eta, k) u^{\prime *}(\eta, k)-u^{*}(\eta, k) u^{\prime}(\eta, k)=\frac{i}{a^{D-2}(\eta)} .
$$

Using Eq. (30), Eqs. (27) and (29) can be solved for $\alpha(\vec{k})$ as

$$
\alpha(\vec{k})=-i a^{D-2}\left(\eta_{I}\right)\left[u^{\prime *}\left(\eta_{I}, k\right) \widetilde{\varphi}\left(\eta_{I}, \vec{k}\right)-u^{*}\left(\eta_{I}, k\right) \widetilde{\varphi}^{\prime}\left(\eta_{I}, \vec{k}\right)\right] .
$$

The creation operator $\alpha^{\dagger}(\vec{k})$ is attained by conjugation.

The coefficient $\mathcal{A}$ in solution (21) can be obtained by imposing Wronskian (30) to Eq. (21) and using the power series expansion of the Hankel function $\mathcal{H}_{\nu}^{(1)}$. The result is

$$
u(\eta, k)=\sqrt{\frac{\pi}{4 H}} a^{-\frac{D-1}{2}} \mathcal{H}_{\nu}^{(1)}\left(\frac{k}{H a}\right),
$$

where we used the fact that $H_{\nu}^{(1)}$ is an even function. The Bunch-Davies mode function $u(\eta, k)$ take a particularly simple form in $D=4$,

$$
\left.u(\eta, k)\right|_{D=4}=\frac{H}{\sqrt{2 k^{3}}}(1+i k \eta) e^{-i k \eta}=\frac{H}{\sqrt{2 k^{3}}}\left(1-\frac{i k}{H a}\right) \exp \left[\frac{i k}{H a}\right] .
$$

The inverse Fourier transform of mode solution (27) yields the usual free field expansion

$$
\varphi_{0}(\eta, \vec{x})=\int \frac{d^{D-1} k}{(2 \pi)^{D-1}}\left\{u(\eta, k) e^{i \vec{k} \cdot \vec{x}} \alpha(\vec{k})+u^{*}(\eta, k) e^{-i \vec{k} \cdot \vec{x}} \alpha^{\dagger}(\vec{k})\right\} .
$$

For $D=4$, we use Eq. (33) in Eq. (34) and obtain

$$
\varphi_{0}(\eta, \vec{x})=\int \frac{d^{3} k}{(2 \pi)^{3}} \frac{H}{\sqrt{2 k}}\left\{e^{-i k \eta+i \vec{k} \cdot \vec{x}}\left(\frac{1}{k}+i \eta\right) \alpha(\vec{k})+e^{i k \eta-i \vec{k} \cdot \vec{x}}\left(\frac{1}{k}-i \eta\right) \alpha^{\dagger}(\vec{k})\right\} .
$$


In this paper, we calculate the one- and two-loop corrections to the Bunch-Davies mode function (33) for a MMC scalar with a quartic self interaction, and then, employ it correct the scalar's scale-free tree-order power spectrum $\Delta_{\varphi}^{2}(t, k)$ at one- and two-loop orders. As was mentioned earlier, quantum processes generate effective self-mass which modifies Eq. (11) and, thus, the amplitude $u(\eta, k)$ of the solution gets quantum corrections. How field equation (11) is modified at one- and two-loop orders and how $u(\eta, k)$ gets order- $\lambda$ and $-\lambda^{2}$ corrections are discussed in the next two sections.

\section{LINEARIZED SCHWINGER-KELDISH EFFECTIVE FIELD EQUATION}

For a classically massive scalar field $\phi(x)$, the mass term enters Eq. (11) as

$$
\partial_{\mu}\left(\sqrt{-g} g^{\mu \nu} \partial_{\nu} \phi(x)\right)=m_{0}^{2} \sqrt{-g} \phi(x)
$$

Exactly the same procedure for solving Eq. (11), outlined in Sec. II, yields the Bunch-Davies mode function for a massive scalar. It has the form given in Eq. (32) with $\nu=\sqrt{\left(\frac{D-1}{2}\right)^{2}-\frac{m_{0}^{2}}{H^{2}}}$. Quantum induced scalar self-mass-squared, however, enters [7] the linearized SchwingerKeldish effective field equation as a source term integrated against the scalar,

$$
\partial_{\mu}\left(\sqrt{-g} g^{\mu \nu} \partial_{\nu} \varphi_{0}(x)\right)=\int_{\eta_{i}}^{0} d \eta^{\prime} \int d^{3} x^{\prime} M^{2}\left(x ; x^{\prime}\right) \varphi_{0}\left(x^{\prime}\right),
$$

where we have taken the unregulated limit $D=4$ after renormalizing the scalar self masssquared. The Schwinger-Keldish formalism [16] is a covariant extension of the Feynman's formulation which produces expectation values rather than in-out matrix elements. The reviews in Ref. [17] explain the use of the formalism.

The self mass squared can be expressed as a series in powers of the loop counting parameter $\lambda$,

$$
M^{2}\left(x ; x^{\prime}\right)=\sum_{\ell=0}^{\infty} \lambda^{\ell} \mathcal{M}_{\ell}^{2}\left(x ; x^{\prime}\right) .
$$

The tree-order term in the sum

$$
\mathcal{M}_{0}^{2}\left(x ; x^{\prime}\right)=0
$$

since the scalar is classically massless. We calculated the one- and two-loop terms $\mathcal{M}_{1}^{2}\left(x ; x^{\prime}\right)$ and $\mathcal{M}_{2}^{2}\left(x ; x^{\prime}\right)$ in Ref. [7]. The one-loop term is simple

$$
\mathcal{M}_{1}^{2}\left(x ; x^{\prime}\right)=\frac{H^{2}}{8 \pi^{2}} a^{4} \ln (a) \delta^{4}\left(x-x^{\prime}\right) .
$$


The two-loop term consists of six terms

$$
\mathcal{M}_{2}^{2}\left(x ; x^{\prime}\right) \equiv \sum_{n=1}^{6} \mathcal{M}_{2, n}^{2}\left(x ; x^{\prime}\right)
$$

where

$$
\begin{aligned}
& \mathcal{M}_{2,1}^{2}\left(x ; x^{\prime}\right)=\frac{i}{2^{12} 3 \pi^{6}} a a^{\prime} \partial^{4}\left[\frac{\ln \left(\mu^{2} \Delta x_{++}^{2}\right)}{\Delta x_{++}^{2}}-\frac{\ln \left(\mu^{2} \Delta x_{+-}^{2}\right)}{\Delta x_{+-}^{2}}\right] \\
& \mathcal{M}_{2,2}^{2}\left(x ; x^{\prime}\right)=-\frac{i H^{2}}{2^{9} \pi^{6}}\left(a a^{\prime}\right)^{2} \partial^{2}\left[\ln \left(\frac{H e^{\frac{3}{4}}}{2 \mu}\right)\left(\frac{\ln \left(\mu^{2} \Delta x_{++}^{2}\right)}{\Delta x_{++}^{2}}-\frac{\ln \left(\mu^{2} \Delta x_{+-}^{2}\right)}{\Delta x_{+-}^{2}}\right)\right] \\
& \mathcal{M}_{2,3}^{2}\left(x ; x^{\prime}\right)=-\frac{i H^{2}}{2^{11} \pi^{6}}\left(a a^{\prime}\right)^{2} \partial^{2}\left[\frac{\ln ^{2}\left(\mu^{2} \Delta x_{++}^{2}\right)}{\Delta x_{++}^{2}}-\frac{\ln ^{2}\left(\mu^{2} \Delta x_{+-}^{2}\right)}{\Delta x_{+-}^{2}}\right] \\
& \mathcal{M}_{2,4}^{2}\left(x ; x^{\prime}\right)=-\frac{i H^{4}}{2^{9} \pi^{6}}\left(a a^{\prime}\right)^{3}\left[\frac{\ln ^{2}\left(\frac{\sqrt{e}}{4} H^{2} \Delta x_{++}^{2}\right)}{\Delta x_{++}^{2}}-\frac{\ln ^{2}\left(\frac{\sqrt{e}}{4} H^{2} \Delta x_{+-}^{2}\right)}{\Delta x_{+-}^{2}}\right] \\
& \mathcal{M}_{2,5}^{2}\left(x ; x^{\prime}\right)=\frac{i H^{6}}{2^{10} 3 \pi^{6}}\left(a a^{\prime}\right)^{4}\left[\ln 3\left(\frac{\sqrt{e}}{4} H^{2} \Delta x_{++}^{2}\right)-\ln ^{3}\left(\frac{\sqrt{e}}{4} H^{2} \Delta x_{+-}^{2}\right)\right] \\
& \mathcal{M}_{2,6}^{2}\left(x ; x^{\prime}\right)=-\frac{1}{2^{9} 3 \pi^{4}} a^{2}\left\{\ln (a) \partial^{2}-(2 \ln (a)+1) H a \partial_{0}\right\} \delta^{4}\left(x-x^{\prime}\right) \\
& -\frac{H^{2}}{2^{7} 3^{2} \pi^{4}} a^{4}\left\{4 \ln ^{3}(a)+\frac{23}{2} \ln ^{2}(a)-\left[39+27 \ln \left(\frac{H}{2 \mu}\right)-2 \pi^{2}\right] \ln (a)\right\} \delta^{4}\left(x-x^{\prime}\right) \\
& +\frac{H^{2}}{2^{7} \pi^{4}} a^{4}\left\{\frac{a^{-3}}{81}-\sum_{n=1}^{\infty} \frac{(n+5)}{(n+1)^{3}} a^{-(n+1)}+4 \sum_{n=1}^{\infty} \frac{a^{-(n+2)}}{(n+2)^{3}}+4 \sum_{n=1}^{\infty} \frac{a^{-(n+3)}}{n(n+3)^{3}}\right\} \delta^{4}\left(x-x^{\prime}\right) .
\end{aligned}
$$

In Eqs. (42)-(47) the coordinate intervals $\Delta x_{++}^{2}, \Delta x_{+-}^{2}, \Delta x_{-+}^{2}$ and $\Delta x_{--}^{2}$ are defined as

$$
\begin{aligned}
& \Delta x_{++}^{2}\left(x ; x^{\prime}\right) \equiv r^{2}-\left(\left|\eta-\eta^{\prime}\right|-i \delta\right)^{2}, \\
& \Delta x_{+-}^{2}\left(x ; x^{\prime}\right) \equiv r^{2}-\left(\eta-\eta^{\prime}+i \delta\right)^{2}=\left(\Delta x_{-+}^{2}\left(x ; x^{\prime}\right)\right)^{*}, \\
& \Delta x_{--}^{2}\left(x ; x^{\prime}\right)=\left(\Delta x_{++}^{2}\left(x ; x^{\prime}\right)\right)^{*},
\end{aligned}
$$

where $\vec{r} \equiv \vec{x}-\vec{x}^{\prime}$ and $\delta$ is an infinitesimal positive real parameter.

The quantum corrected scalar mode function $\Phi(x ; \vec{k})$ is the solution of the linearized effective field equation (37),

$$
\partial_{\mu}\left(\sqrt{-g} g^{\mu \nu} \partial_{\nu} \Phi(x ; \vec{k})\right)=\int_{\eta_{i}}^{0} d \eta^{\prime} \int d^{3} x^{\prime}\left\{\sum_{\ell=0}^{\infty} \lambda^{\ell} \mathcal{M}_{\ell}^{2}\left(x ; x^{\prime}\right)\right\} \Phi\left(x^{\prime} ; \vec{k}\right) .
$$

We solve the effective field equation perturbatively. Expanding the plane wave solution $\Phi(\eta, k)$ in powers $\lambda$

$$
\Phi(x ; \vec{k}) \equiv \Phi(\eta ; k) e^{i \vec{k} \cdot \vec{x}} \equiv \sum_{\ell=0}^{\infty} \lambda^{\ell} \Phi_{\ell}(\eta, k) e^{i \vec{k} \cdot \vec{x}}
$$


and substituting it back into Eq. (51), we find

$$
a^{2}\left[\partial_{0}^{2}+2 H a \partial_{0}+k^{2}\right] \Phi_{\ell}(\eta, k)=-\sum_{n=0}^{\ell} \int_{\eta_{i}}^{0} d \eta^{\prime} \int d^{3} x^{\prime} \mathcal{M}_{n}^{2}\left(x ; x^{\prime}\right) \Phi_{\ell-n}\left(\eta^{\prime}, k\right) e^{i \vec{k} \cdot\left(\vec{x}^{\prime}-\vec{x}\right)} .
$$

Note that the tree-order $(\ell=0)$ solution $\Phi_{0}(\eta, k)$ is the Bunch-Davies mode function $u(\eta, k)$ given in Eq. (33). Converting Eq. (53) for $\Phi_{\ell}$ from conformal time $\eta$ to comoving time $t=-\ln (-H \eta) / H$ yields

$$
\left[\frac{\partial^{2}}{\partial t^{2}}+3 H \frac{\partial}{\partial t}+\frac{k^{2}}{a^{2}}\right] \Phi_{\ell}=-\frac{1}{a^{4}} \sum_{n=0}^{\ell} \int_{\eta_{i}}^{0} d \eta^{\prime} \int d^{3} x^{\prime} \mathcal{M}_{n}^{2}\left(x ; x^{\prime}\right) \Phi_{\ell-n}\left(\eta^{\prime}, k\right) e^{i \vec{k} \cdot\left(\vec{x}^{\prime}-\vec{x}\right)} .
$$

Next, we solve this second order, linear, non-homogeneous integro-differential equation to get the one- and two-loop quantum corrections to the Bunch-Davies mode function.

\section{QUANTUM-CORRECTED SCALAR MODE FUNCTION}

The scalar self mass-squared is known [7] at one- and two-loop orders only. Hence, we can solve Eq. (54) for $\Phi_{1}(\eta, k)$ and $\Phi_{2}(\eta, k)$ by applying the Green's function technique.

\section{A. Order $\lambda$ Correction $\Phi_{1}(\eta, k)$ for the Scalar Mode Function}

Using Eqs. (39) and (40) in Eq. (54), one obtains the integro-differential equation for the order $\lambda$ correction $\Phi_{1}(\eta, \vec{k})$,

$$
\begin{aligned}
{\left[\frac{\partial^{2}}{\partial t^{2}}+3 H \frac{\partial}{\partial t}+\frac{k^{2}}{a^{2}}\right] \Phi_{1} } & =-\frac{1}{a^{4}} \int_{\eta_{i}}^{0} d \eta^{\prime} u\left(\eta^{\prime}, k\right) \int d^{3} x^{\prime} \mathcal{M}_{1}^{2}\left(x ; x^{\prime}\right) e^{-i \vec{k} \cdot\left(\vec{x}-\vec{x}^{\prime}\right)} \\
& =-\frac{H^{2}}{8 \pi^{2}} u(\eta, k) \ln (a)
\end{aligned}
$$

The solution $\Phi_{1}(\eta, k)$ of Eq. (55) can be written as an integral over comoving time as

$$
\Phi_{1}(\eta, k)=-\frac{H^{2}}{8 \pi^{2}} \int_{0}^{t} d t^{\prime} G\left(t, t^{\prime} ; k\right) u\left(\eta^{\prime}, k\right) \ln \left(a\left(\eta^{\prime}\right)\right)
$$

where the Green's function

$$
G\left(t, t^{\prime} ; k\right)=\frac{\theta\left(t-t^{\prime}\right)}{W\left(t^{\prime}, k\right)}\left[u(\eta, k) u^{*}\left(\eta^{\prime}, k\right)-u^{*}(\eta, k) u\left(\eta^{\prime}, k\right)\right] .
$$

In Eq. (57), $\theta\left(t-t^{\prime}\right)$ is the Heaviside step function, and $W\left(t^{\prime}, k\right)$ is the Wronskian

$$
W\left(t^{\prime}, k\right)=\dot{u}\left(\eta^{\prime}, k\right) u^{*}\left(\eta^{\prime}, k\right)-u\left(\eta^{\prime}, k\right) \dot{u}^{*}\left(\eta^{\prime}, k\right)=\frac{-i}{a^{3}\left(\eta^{\prime}\right)}=i H^{3} \eta^{\prime 3}=-i e^{-3 H t^{\prime}}
$$


where a dot denotes derivative with respect to comoving time $t$. Making the change of variable $t^{\prime}=\ln \left(a\left(\eta^{\prime}\right)\right) / H$ and using Eqs. (33), (57) and (58) in Eq. (56), we find

$$
\begin{aligned}
& \Phi_{1}(\eta, k)=-\frac{i H}{8 \pi^{2}} \int_{1}^{a} d a^{\prime} a^{\prime 2} \ln \left(a^{\prime}\right)\left[u(\eta, k)\left|u\left(\eta^{\prime}, k\right)\right|^{2}-u^{*}(\eta, k) u^{2}\left(\eta^{\prime}, k\right)\right] \\
& =-\frac{i H^{3}}{16 \pi^{2} k^{3}}\left\{u(\eta, k) \int_{1}^{a} d a^{\prime} \ln \left(a^{\prime}\right)\left[a^{\prime 2}+\frac{k^{2}}{H^{2}}\right]-u^{*}(\eta, k) \int_{1}^{a} d a^{\prime} \ln \left(a^{\prime}\right)\left[a^{\prime}-\frac{i k}{H}\right]^{2} e^{\frac{2 i k}{H a^{\prime}}}\right\} .
\end{aligned}
$$

Evaluating the integrals in Eq. (60) gives the one-loop correction to the mode function

$$
\begin{aligned}
& \Phi_{1}(\eta, k)=-\frac{i H^{3}}{16 \pi^{2} k^{3}}\left\{u(\eta, k)\left[a^{3} \frac{\ln (a)}{3}-\frac{a^{3}}{9}+\frac{1}{9}+\frac{k^{2}}{H^{2}}(a \ln (a)-a+1)\right]\right. \\
& -u^{*}(\eta, k)\left[\frac{i k^{3}}{H^{3}} \frac{\ln ^{2}(a)}{3}+\sum_{n \doteq 0}^{\infty} \frac{1}{n !}\left(\frac{2 i k}{H}\right)^{n}\left(\left[\frac{a^{3-n}}{3-n} \ln (a)-\frac{\left(a^{3-n}-1\right)}{(3-n)^{2}}\right]\right.\right. \\
& \left.\left.\left.-\frac{2 i k}{H}\left[\frac{a^{2-n}}{2-n} \ln (a)-\frac{\left(a^{2-n}-1\right)}{(2-n)^{2}}\right]-\frac{k^{2}}{H^{2}}\left[\frac{a^{1-n}}{1-n} \ln (a)-\frac{\left(a^{1-n}-1\right)}{(1-n)^{2}}\right]\right)\right]\right\},
\end{aligned}
$$

where we use, in the sum, the $\doteq$ symbol to indicate that the terms that diverge for each $n$, as $n$ runs from 0 to $\infty$, are to be excluded. In other words, the terms in the first square brackets for $n=3$, the terms in the second square brackets for $n=2$ and the terms in the third square brackets for $n=1$ are to be excluded in the sum. Equation (61) is an exact result for $\Phi_{1}(\eta, k)$. If we express it in powers of $k / H$, we see that the lowest order $k$-dependent correction comes in quadratic order

$$
\begin{array}{r}
\Phi_{1}(\eta, k)=\frac{u(0, k)}{2^{4} 3 \pi^{2}}\left\{-\ln ^{2}(a)+\frac{2 \ln (a)}{3}-\frac{2}{9}+\frac{2 a^{-3}}{9}-\frac{k^{2}}{H^{2}}\left[\frac{1}{5}+\frac{\ln ^{2}(a)}{2 a^{2}}-\frac{7 \ln (a)}{3 a^{2}}+\frac{16}{9 a^{2}}-\frac{2}{a^{3}}+\frac{a^{-5}}{45}\right]\right. \\
-\frac{i k^{3}}{H^{3}}\left[\frac{2}{27}-\frac{\ln ^{2}(a)}{3 a^{3}}-\frac{2 \ln (a)}{9 a^{3}}-\frac{2 a^{-3}}{27}\right]+\frac{k^{4}}{H^{4}}\left[\frac{3}{140}-\frac{a^{-2}}{10}+\frac{\ln ^{2}(a)}{8 a^{4}}-\frac{\ln (a)}{12 a^{4}}+\frac{5 a^{-4}}{18}-\frac{a^{-5}}{5}+\frac{a^{-7}}{1260}\right] \\
\left.+\mathcal{O}\left(i k^{5} / H^{5}\right)\right\} .
\end{array}
$$

Note that, leading logarithm terms in the $k$-independent part of this result agrees with our putative late time solution (1). The $k$-dependent shifts are intrinsic to complete solution (61). The $k$-independent shift $-2 / 9$ in Eq. (62), which is also time independent, can be absorbed into a field strength renormalization (5), and hence it is not an observable. To see this, notice that the full mode function (52) becomes $\bar{\Phi}(x ; k)=\bar{\Phi}(\eta, k) e^{i \vec{k} \cdot \vec{x}}=[\Phi(\eta ; k) / \sqrt{Z}] e^{i \vec{k} \cdot \vec{x}}$, if we define a new field $\bar{\varphi}(x) \equiv \varphi(x) / \sqrt{Z}$. Expressing $\sqrt{Z}$ in powers of $\lambda$ as $\sqrt{Z}=1+\lambda Z_{1}+$ $\lambda^{2} Z_{2}+\mathcal{O}\left(\lambda^{3}\right)$, we find

$$
\begin{aligned}
& \bar{\Phi}(\eta, k)=\sum_{\ell=0}^{\infty} \lambda^{\ell} \bar{\Phi}_{\ell}(\eta, k)=\frac{1}{\sqrt{Z}} \sum_{\ell=0}^{\infty} \lambda^{\ell} \Phi_{\ell}(\eta, k) \\
& \quad=u(\eta, k)+\lambda\left[\Phi_{1}(\eta, k)-Z_{1} u(\eta, k)\right]+\lambda^{2}\left[\Phi_{2}(\eta, k)-Z_{1} \Phi_{1}(\eta, k)-Z_{2} u(\eta, k)\right]+\mathcal{O}\left(\lambda^{3}\right) .
\end{aligned}
$$


The $k$ - and $\eta$-independent correction in Eq. (62) has the form $\lambda u(0, k) Z_{1}$. Except for the fact that $\eta$ has been taken to 0 , that is exactly the same as a field strength renormalization. Because $u(\eta, k)$ approaches a nonzero constant in the late time limit $\eta \rightarrow 0$, and also because we can unambiguously determine only the late time behavior of $\Phi_{1}(\eta, k)$ (the initial state hasn't been perturbatively corrected), we conclude that, if $Z_{1}=-1 /\left(2^{3} 3^{3} \pi^{2}\right)$, a $k$-independent constant shift would not be present in the $\bar{\Phi}_{1}(\eta, k)$.

The $k$-dependent constant shifts in Eq. (62), on the other hand, cannot be absorbed with a field strength renormalization and they are, in principle, physical. To fix them and the terms that redshift like inverse powers of the scale factor in $\Phi_{1}(\eta, k)$ unambiguously, however, one must compute the first order term $\lambda\left|\Omega_{1}\right\rangle$ in the initial state correction

$$
|\Delta \Omega\rangle \equiv \sum_{n=1}^{\infty} \lambda^{n}\left|\Omega_{n}\right\rangle
$$

as in Ref. [6], and use it to solve the effective field equation. The terms which grow like powers of $\ln (a)$ in the one-loop correction $\Phi_{1}(\eta, k)$ represent the effect of inflationary particle production pushing the field up its quartic potential and are physical, in principle. We compute the two-loop correction $\Phi_{2}(\eta, k)$, in the next section.

\section{B. Order $\lambda^{2}$ Correction $\Phi_{2}(\eta, k)$ for the Scalar Mode Function}

Using Eq. (54), the integro-differential equation for $\Phi_{2}(\eta, k)$ is obtained as

$$
\left[\frac{\partial^{2}}{\partial t^{2}}+3 H \frac{\partial}{\partial t}+\frac{k^{2}}{a^{2}}\right] \Phi_{2}=\frac{-1}{a^{4}} \int_{\eta_{i}}^{0} d \eta^{\prime} \int d^{3} x^{\prime}\left\{\mathcal{M}_{1}^{2}\left(x ; x^{\prime}\right) \Phi_{1}\left(\eta^{\prime}, k\right)+\mathcal{M}_{2}^{2}\left(x ; x^{\prime}\right) u\left(\eta^{\prime}, k\right)\right\} e^{-i \vec{k} \cdot\left(\vec{x}-\vec{x}^{\prime}\right)} .
$$

The right hand side has contributions due to both one- and two-loop self-mass-squared terms. The contribution due to one-loop mass-squared term is trivial to integrate. Using Eqs. (40) one finds

$$
-\frac{1}{a^{4}} \int_{\eta_{i}}^{0} d \eta^{\prime} \int d^{3} x^{\prime} \mathcal{M}_{1}^{2}\left(x ; x^{\prime}\right) \Phi_{1}\left(\eta^{\prime}, k\right) e^{-i \vec{k} \cdot\left(\vec{x}-\vec{x}^{\prime}\right)}=-\frac{H^{2}}{8 \pi^{2}} \ln (a) \Phi_{1}(\eta, k),
$$

where $\Phi_{1}(\eta, k)$ is given in Eqs. (61) and (62). It is useful to break the contribution due to the two-loop self-mass-squared term into a sum of six integrals:

$$
-\frac{1}{a^{4}} \int_{\eta_{i}}^{0} d \eta^{\prime} \int d^{3} x^{\prime} \mathcal{M}_{2}^{2}\left(x ; x^{\prime}\right) u\left(\eta^{\prime}, k\right) e^{-i \vec{k} \cdot\left(\vec{x}-\vec{x}^{\prime}\right)} \equiv \sum_{n=1}^{6} \mathcal{I}_{n}(\eta, k)
$$


where

$$
\mathcal{I}_{n}(\eta, k) \equiv-\frac{1}{a^{4}} \int_{\eta_{i}}^{0} d \eta^{\prime} \int d^{3} x^{\prime} \mathcal{M}_{2, n}^{2}\left(x ; x^{\prime}\right) u\left(\eta^{\prime}, k\right) e^{-i \vec{k} \cdot\left(\vec{x}-\vec{x}^{\prime}\right)} .
$$

Recall that $\mathcal{M}_{2, n}^{2}\left(x ; x^{\prime}\right)$ are given in Eqs. (42)-(47). Thus, Eq. (65) for $\Phi_{2}$ can be written as

$$
\left[\frac{\partial^{2}}{\partial t^{2}}+3 H \frac{\partial}{\partial t}+\frac{k^{2}}{a^{2}}\right] \Phi_{2}=\mathcal{S}(\eta, k)
$$

where we define the non-homogeneous (source) term of the differential equation as

$$
\mathcal{S}(\eta, k) \equiv-\frac{H^{2}}{8 \pi^{2}} \ln (a(\eta)) \Phi_{1}(\eta, k)+\sum_{n=1}^{6} \mathcal{I}_{n}(\eta, k)
$$

We explicitly evaluate the first integral in the source term,

$$
\mathcal{I}_{1}(\eta, k)=\frac{-i}{2^{12} 3 \pi^{6} a^{3}} \int d^{4} x^{\prime} a^{\prime}\left\{\partial^{4}\left[\frac{\ln \left(\mu^{2} \Delta x_{++}^{2}\right)}{\Delta x_{++}^{2}}-\frac{\ln \left(\mu^{2} \Delta x_{+-}^{2}\right)}{\Delta x_{+-}^{2}}\right]\right\} u\left(\eta^{\prime}, k\right) e^{-i \vec{k} \cdot\left(\vec{x}-\vec{x}^{\prime}\right)}
$$

to illustrate the relevant calculation techniques in this section, and outline the key steps for the remaining five in the Appendices. The first step is to extract another derivative making use of the identity

$$
\frac{\ln \left(\mu^{2} \Delta x^{2}\right)}{\Delta x^{2}}=\frac{\partial^{2}}{8}\left[\ln ^{2}\left(\mu^{2} \Delta x^{2}\right)-2 \ln \left(\mu^{2} \Delta x^{2}\right)\right] .
$$

Then, one exploits the cancelation between the ++ and +- terms except within the past light-cone, using

$$
\ln \left[\mu^{2} \Delta x_{+ \pm}^{2}\right]=\ln \left[\mu^{2}\left(\Delta \eta^{2}-r^{2}\right)\right] \pm i \pi \theta\left(\Delta \eta^{2}-r^{2}\right)
$$

The result is

$$
\begin{aligned}
\mathcal{I}_{1}(\eta, k) & =\frac{-i e^{-i \vec{k} \cdot \vec{x}}}{2^{15} 3 \pi^{6} a^{3}} \int d^{4} x^{\prime} a^{\prime}\left\{\partial^{6}\left[\begin{array}{c}
\ln ^{2}\left(\mu^{2} \Delta x_{++}^{2}\right)-2 \ln \left(\mu^{2} \Delta x_{++}^{2}\right) \\
-\ln ^{2}\left(\mu^{2} \Delta x_{+-}^{2}\right)+2 \ln \left(\mu^{2} \Delta x_{+-}^{2}\right)
\end{array}\right]\right\} u\left(\eta^{\prime}, k\right) e^{i \vec{k} \cdot \vec{x}^{\prime}} \\
& =\frac{e^{-i \vec{k} \cdot \vec{x}}}{2^{13} 3 \pi^{5}} \frac{\partial^{6}}{a^{3}} \int d \eta^{\prime} a^{\prime} u\left(\eta^{\prime}, k\right) \int d^{3} x^{\prime} e^{i \vec{k} \cdot \vec{x}^{\prime}} \theta(\Delta \eta-r)\left\{\ln \left[\mu^{2}\left(\Delta \eta^{2}-r^{2}\right)\right]-1\right\} .
\end{aligned}
$$

The next step is to perform the angular integrations using

$$
\int d^{3} x^{\prime} e^{i \vec{k} \cdot \vec{x}^{\prime}} f(\Delta x)=4 \pi e^{i \vec{k} \cdot \vec{x}} \int_{0}^{\infty} d r r^{2} \frac{\sin (k r)}{k r} f(r) .
$$

We can also pass the spatial phase factor through the derivative,

$$
\partial^{6} e^{i \vec{k} \cdot \vec{x}}=-e^{i \vec{k} \cdot \vec{x}}\left(\partial_{0}^{2}+k^{2}\right)^{3}
$$


to reach the form,

$$
\mathcal{I}_{1}(\eta, k)=\frac{-1}{2^{11} 3 \pi^{4}} \frac{\left(\partial_{0}^{2}+k^{2}\right)^{3}}{a^{3} k} \int_{\eta_{i}}^{\eta} d \eta^{\prime} a^{\prime} u\left(\eta^{\prime}, k\right) \int_{0}^{\Delta \eta} d r r \sin (k r)\left\{\ln \left[\mu^{2}\left(\Delta \eta^{2}-r^{2}\right)\right]-1\right\} .
$$

The radial integration in Eq. (78) involves a combination of special functions. First, we make the change of variable, $r \equiv \Delta \eta z \equiv \frac{\alpha}{k} z$, and obtain

$$
\begin{aligned}
\int_{0}^{\Delta \eta} d r r \sin (k r)\left\{\ln \left[\mu^{2}\left(\Delta \eta^{2}-r^{2}\right)\right]-1\right\} \\
=\Delta \eta^{2} \int_{0}^{1} d z z \sin (k \Delta \eta z)\left\{2 \ln (\mu \Delta \eta)-1+\ln \left(1-z^{2}\right)\right\} \\
=\frac{1}{k^{2}}[\sin (k \Delta \eta)-k \Delta \eta \cos (k \Delta \eta)][2 \ln (\mu \Delta \eta)-1]+\Delta \eta^{2} \xi(k \Delta \eta)
\end{aligned}
$$

where we define

$$
\begin{aligned}
\xi(k \Delta \eta)= & \xi(\alpha) \equiv \int_{0}^{1} d z z \sin (\alpha z) \ln \left(1-z^{2}\right) \\
= & \frac{2}{\alpha^{2}} \sin (\alpha)-\frac{1}{\alpha^{2}}[\cos (\alpha)+\alpha \sin (\alpha)]\left[\operatorname{si}(2 \alpha)+\frac{\pi}{2}\right] \\
& +\frac{1}{\alpha^{2}}[\sin (\alpha)-\alpha \cos (\alpha)]\left[\operatorname{ci}(2 \alpha)-\ln \left(\frac{\alpha}{2}\right)-\gamma\right] .
\end{aligned}
$$

See Appendix A for the evaluation of $\xi(\alpha)$. The sine and cosine integrals are defined in Eqs. (A5) and (A6), respectively. Inserting Eq. (80) into Eq. (78) and applying the derivatives using

$$
\partial_{0} \int_{\eta_{i}}^{\eta} d \eta^{\prime} f\left(\eta, \eta^{\prime}\right)=f(\eta, \eta)+\int_{\eta_{i}}^{\eta} d \eta^{\prime} \frac{\partial f\left(\eta, \eta^{\prime}\right)}{\partial \eta}
$$

we obtain

$$
\begin{aligned}
\mathcal{I}_{1}(\eta, k)=\frac{-1}{2^{9} 3 \pi^{4}} \frac{k^{2}}{a^{3}}\{a u(\eta, k)+k & \int_{\eta_{i}}^{\eta} d \eta^{\prime} a^{\prime} u\left(\eta^{\prime}, k\right)\left[\frac{\cos (\alpha)}{\alpha}-\frac{\sin (\alpha)}{\alpha^{2}}+\ln (2 \mu \Delta \eta) \sin (\alpha)\right] \\
& \left.+\left[2 \partial_{0}+\frac{\partial_{0}^{3}}{k^{2}}\right] \int_{\eta_{i}}^{\eta} d \eta^{\prime} a^{\prime} u\left(\eta^{\prime}, k\right) \ln (2 \mu \Delta \eta) \cos (\alpha)\right\} .
\end{aligned}
$$

Expanding the logarithm,

$$
\ln (2 \mu \Delta \eta)=\ln \left(\frac{2 \mu}{H a^{\prime}}\right)+\ln \left(1-\frac{a^{\prime}}{a}\right)=\ln \left(\frac{2 \mu}{H a^{\prime}}\right)-\sum_{n=1}^{\infty} \frac{a^{\prime n}}{n a^{n}}
$$

evaluating the integrals, and taking the derivatives using $\partial_{0}=\partial_{\eta}=H a^{2} \partial_{a}$ one can express $\mathcal{I}_{1}(\eta, k)$ in powers of $k / a$ as

$$
\mathcal{I}_{1}(\eta, k)=\frac{-1}{2^{9} 3 \pi^{4}} \frac{H}{\sqrt{2 k^{3}}}\left\{H^{2}\left[2 \ln \left(\frac{2 \mu}{H a}\right)-3-\sum_{n=2}^{\infty} \frac{(n-1) n a^{-(n+1)}}{n+1}\right]+\frac{k^{2}}{a^{2}}\left[\ln \left(\frac{2 \mu}{H a}\right)-\frac{1}{2}\right.\right.
$$




$$
\begin{aligned}
& \left.-\frac{1}{2} \sum_{n=0}^{\infty} \frac{\left(n^{2}+3 n+4\right) a^{-(n+1)}}{n+1}\right]+\frac{i k^{3}}{H a^{3}}\left[\frac{2}{3} \ln \left(\frac{2 \mu}{H}\right)-1-\frac{1}{3} \sum_{n=0}^{\infty} \frac{(n+2)(n+3) a^{-(n+1)}}{n+1}\right] \\
& \left.-\frac{k^{4}}{H^{2} a^{4}}\left[\frac{1}{4} \ln \left(\frac{2 \mu}{H a}\right)-\frac{7}{8}-\frac{1}{8} \sum_{n=0}^{\infty} \frac{\left(n^{2}+7 n+8\right) a^{-(n+1)}}{n+1}\right]+\mathcal{O}\left(\frac{i k^{5}}{H^{3}}\right)\right\} .
\end{aligned}
$$

Calculations of $\mathcal{I}_{n}(\eta, k)$ for $2 \leq n \leq 6$ are given in Appendix B. Using Eqs. (62), (86), (B3), (B12), (B17), (B21), (B23), one obtains an exact expression for the source term $\mathcal{S}(\eta, k)$ of Eq. (69). The result is given in Appendix C.

The solution $\Phi_{2}(\eta, k)$ of Eq. (69) can be written as an integral over comoving time

$$
\Phi_{2}(\eta, k)=\int_{0}^{t} d t^{\prime} G\left(t, t^{\prime} ; k\right) S\left(\eta^{\prime}, k\right) .
$$

Inserting the Green's function $G\left(t, t^{\prime} ; k\right)$ given in Eq. (57) into Eq. (87) yields

$$
\begin{aligned}
\Phi_{2}(\eta, k)=\frac{i}{\sqrt{2 k^{3}}}\left\{u(\eta, k) \int_{1}^{a} d a^{\prime}\right. & {\left[a^{\prime 2}+\frac{i k a^{\prime}}{H}\right] \exp \left(\frac{-i k}{H a^{\prime}}\right) S\left(\eta^{\prime}, k\right) } \\
& \left.-u^{*}(\eta, k) \int_{1}^{a} d a^{\prime}\left[a^{\prime 2}-\frac{i k a^{\prime}}{H}\right] \exp \left(\frac{i k}{H a^{\prime}}\right) S\left(\eta^{\prime}, k\right)\right\} .
\end{aligned}
$$

Using Eq. (C2) in Eq. (88) one finds an exact expression for the two-loop correction $\Phi_{2}(\eta, k)$. If we express it in powers of $k / H$, we see that the lowest order $k$-dependent correction comes in quadratic order

$$
\begin{aligned}
& \Phi_{2}(\eta, k)=\frac{u(0, k)}{2^{8} 3^{3} \pi^{4}}\left\{\frac{11 \ln ^{4}(a)}{2}-\ln ^{3}(a)+\left(27 \ln \left(\frac{2 \mu}{H}\right)-\frac{547}{12}+4 \pi^{2}+\frac{2}{3 a^{3}}\right) \ln ^{2}(a)\right. \\
& +\left(18 \ln ^{2}\left(\frac{2 \mu}{H}\right)-48 \ln \left(\frac{2 \mu}{H}\right)+\frac{817}{18}+\frac{5 \pi^{2}}{3}-48 \zeta(3)+\sum_{p=2}^{\infty} \sum_{n=1}^{p-1} \frac{72}{n(p-n) p(p+3)}+\frac{\frac{43}{18}+\frac{4 \pi^{2}}{3}}{a^{3}}\right) \ln (a) \\
& +\mathcal{C}_{0}+\mathcal{O}\left(a^{-2}\right)+\frac{k^{2}}{H^{2}}\left[\left(\frac{3}{5}+\frac{11 \ln ^{2}(a)}{4 a^{2}}-\frac{45 \ln (a)}{2 a^{2}}+\frac{\frac{27}{2} \ln \left(\frac{2 \mu}{H}\right)+\frac{1181}{24}+2 \pi^{2}}{a^{2}}+\frac{6}{a^{3}}-\frac{a^{-5}}{15}\right) \ln ^{2}(a)\right. \\
& +\left(\frac{12983}{750}-\frac{4 \pi^{2}}{5}+\frac{9 \ln ^{2}\left(\frac{2 \mu}{H}\right)-78 \ln \left(\frac{2 \mu}{H}\right)-\frac{2705}{36}-\frac{43 \pi^{2}}{6}-24 \zeta(3)}{a^{2}}+\sum_{p=2}^{\infty} \sum_{n=1}^{p-1} \frac{36 a^{-2}}{n(p-n) p(p+3)}\right. \\
& \left.\left.-\frac{\frac{151}{2}-4 \pi^{2}}{a^{3}}-\frac{\frac{263}{900}+\frac{2 \pi^{2}}{15}}{a^{5}}\right) \ln (a)+\mathcal{C}_{2}+\mathcal{O}\left(a^{-2}\right)\right]+\frac{i k^{3}}{H^{3}}\left[\left(\frac{2}{9}+\frac{\ln ^{2}(a)}{2 a^{3}}+\frac{13 \ln (a)}{9 a^{3}}\right.\right. \\
& \left.-\frac{9 \ln \left(\frac{2 \mu}{H}\right)+\frac{1921}{252}}{a^{3}}\right) \ln ^{2}(a)+\left(\frac{457}{54}-\frac{4 \pi^{2}}{9}-\frac{6 \ln ^{2}\left(\frac{2 \mu}{H}\right)-4 \ln \left(\frac{2 \mu}{H}\right)-\frac{5051}{189}-\frac{13 \pi^{2}}{3}+16 \zeta(3)}{a^{3}}\right. \\
& \left.\left.+\sum_{p=4}^{\infty} \sum_{n=1}^{p-1} \frac{12(p+4) a^{-3}}{n(p-n)(p-3)(p-2) p}\right) \ln (a)+\mathcal{C}_{3}+\mathcal{O}\left(a^{-2}\right)\right]-\frac{k^{4}}{H^{4}}\left[\left(\frac{9}{140}-\frac{3}{10 a^{2}}+\frac{11 \ln ^{2}(a)}{16 a^{4}}-\frac{\ln (a)}{8 a^{4}}\right.\right. \\
& \left.+\frac{\frac{27}{8} \ln \left(\frac{2 \mu}{H}\right)+\frac{1109}{96}+\frac{\pi^{2}}{2}}{a^{4}}+\frac{3 a^{-5}}{5}-\frac{a^{-7}}{420}\right) \ln ^{2}(a)+\left(\frac{540817}{171500}-\frac{6 \pi^{2}}{35}-\frac{\frac{11183}{1500}-\frac{2 \pi^{2}}{5}}{a^{2}}+\frac{6}{a^{3}}\right.
\end{aligned}
$$




$$
\begin{aligned}
& +\frac{\frac{9}{4} \ln ^{2}\left(\frac{2 \mu}{H}\right)-6 \ln \left(\frac{2 \mu}{H}\right)+\frac{1033}{144}+\frac{5 \pi^{2}}{24}-6 \zeta(3)}{a^{4}}+\sum_{p=2}^{\infty} \sum_{n=1}^{p-1} \frac{9 a^{-4}}{n(p-n) p(p+3)}-\frac{\frac{707}{100}-\frac{2 \pi^{2}}{5}}{a^{5}} \\
& \left.\left.\left.-\frac{\frac{2081}{176400}+\frac{\pi^{2}}{210}}{a^{7}}\right) \ln (a)+\mathcal{C}_{4}+\mathcal{O}\left(a^{-2}\right)\right]+\mathcal{O}\left(\frac{i k^{5}}{H^{5}}\right)\right\}
\end{aligned}
$$

where $\mathcal{C}_{0}, \mathcal{C}_{2}, \mathcal{C}_{3}$ and $\mathcal{C}_{4}$ are time independent constant terms. The quartic and cubic order logarithm terms in the $k$-independent part of this result agrees with our putative late time solution (1). The $k$-independent shift $\mathcal{C}_{0}$, which is also time independent, can be absorbed into a field strength renormalization and is not an observable. (If $Z_{2}=\left(27 \mathcal{C}_{0}-4\right) /\left(2^{8} 3^{6} \pi^{4}\right)$ in Eq. (63), a $k$-independent constant shift would not be present in the $\bar{\Phi}_{2}$.) To fix the $k$-dependent constant shifts $\mathcal{C}_{2}, \mathcal{C}_{3}, \mathcal{C}_{4}$ and the terms that redshift like inverse powers of the

scale factor unambiguously, one needs to compute the second order term $\lambda^{2}\left|\Omega_{2}\right\rangle$ in initial state correction (64), as in Ref. [6], and use it to solve the effective field equation. The terms in powers of infrared logarithms are physical, in principle.

Thus, the one- and two-loop corrected scalar mode function can now be expressed as

$$
\Phi(x, k)=\Phi(\eta, k) e^{i \vec{k} \cdot \vec{x}}=\left(u(\eta, k)+\lambda \Phi_{1}(\eta, k)+\lambda^{2} \Phi_{2}(\eta, k)+\mathcal{O}\left(\lambda^{3}\right)\right) e^{i \vec{k} \cdot \vec{x}}
$$

where $u(\eta, k), \Phi_{1}(\eta, k)$ and $\Phi_{2}(\eta, k)$ are obtained in Eqs. (33), (61)-(62) and (89), respectively. In general, $k$-dependent physical shifts in a mode function tilt the power spectrum. Assuming we can measure the tree-order power spectrum of our scalar $\varphi(x)$, we expect that the $k$-dependent shifts in the loop corrections $\Phi_{\ell}(\eta, k)$ ought to induce a tilt, which may be observed, in the power spectrum. In Sec. V, we use the one- and two-loop mode function corrections, $\Phi_{1}(\eta, k)$ and $\Phi_{2}(\eta, k)$, obtained in Eqs. (62) and (89), to compute the scalar's power spectrum at one- and two-loop orders.

\section{QUANTUM-CORRECTED POWER SPECTRUM}

The power spectrum $\mathcal{P}(t, k)$ is a fundamental theoretical quantity in cosmology which measures typical amplitude of fluctuations in a field as a function of comoving wave number $k$. It is defined as the Fourier transform of the equal-time two-point correlation function of the field,

$$
\mathcal{P}_{\varphi}(t, k) \equiv \int d^{3} x e^{-i \vec{k} \cdot \vec{x}}\langle\Omega|\varphi(t, \vec{x}) \varphi(t, 0)| \Omega\rangle
$$


The excess power in a bin of size $d k$ centered at $k$ is associated with $\mathcal{P}_{\varphi}(t, k) d^{3} k /(2 \pi)^{3}$. After integrating it over all orientations of $\vec{k}$, an alternative measure of power $\Delta_{\varphi}^{2}(t, k)$ in a mode $k$ is obtained

$$
\frac{k^{2} d k}{2 \pi^{2}} \mathcal{P}_{\varphi}(t, k) \equiv \frac{d k}{k} \Delta_{\varphi}^{2}(t, k) .
$$

Employing the free-field expansion (34) in Eqs. (91) and (92) yields the tree-order power spectrum as

$$
\Delta_{\varphi}^{2}(t, k)=\frac{k^{3}}{2 \pi^{2}} \mathcal{P}_{\varphi}(t, k)=\frac{k^{3}}{2 \pi^{2}}|u(t, k)|^{2},
$$

where $u(t, k)$ is the tree-order mode function given in Eq. (33). Hence,

$$
\Delta_{\varphi}^{2}(t, k)=\frac{H^{2}}{4 \pi^{2}}\left(1+\frac{k^{2}}{H^{2}} a^{-2}\right) .
$$

This yields the well known scale-invariant power spectrum $H^{2} / 4 \pi^{2}$, in the late time limit.

A non-linear generalization of $\Delta_{\varphi}^{2}(t, k)$ is realized by replacing the tree-order BunchDavies mode function $u(t, k)$ in free field expansion (34) with the quantum corrected mode function $\Phi(t, k)$ defined in Eq. (52). Employing this loop corrected field expansion in Eqs. (91) and (92) yields the quantum corrected power spectrum [18] as

$$
\Delta_{\varphi}^{2}(t, k)=\frac{k^{3}}{2 \pi^{2}}|\Phi(t, k)|^{2},
$$

where the amplitude squared for each mode is

$$
\begin{aligned}
|\Phi(t, k)|^{2}=|u(t, k)|^{2} & +\lambda\left[u(t, k) \Phi_{1}^{*}(t, k)+u^{*}(t, k) \Phi_{1}(t, k)\right] \\
& +\lambda^{2}\left[\left|\Phi_{1}(t, k)\right|^{2}+u(t, k) \Phi_{2}^{*}(t, k)+u^{*}(t, k) \Phi_{2}(t, k)\right]+\mathcal{O}\left(\lambda^{3}\right) .
\end{aligned}
$$

Precisely measurable cosmological observables may be sensitive to signals originated from tiny quantum effects. Motivated by the hope to resolve such signals theorists intensively studied - potentially enhanced - loop corrections to inflationary correlators in the recent past [19]. In this section, we calculate the one- and two-loop corrections to the power spectrum of the MMC scalar $\varphi(x)$ with quartic self-interaction during $\Lambda$-driven de Sitter inflation. Inserting Eq. (96) into Eq. (95), and using Eqs. (33), (62) and (89) after field strength renormalization, yields

$$
\begin{aligned}
& \Delta_{\varphi}^{2}(t, k)=\frac{H^{2}}{4 \pi^{2}}\left\{1+\frac{k^{2}}{H^{2}} a^{-2}-\frac{\lambda}{2^{3} 3 \pi^{2}}\left[\ln ^{2}(a)-\frac{2 \ln (a)}{3}-\frac{2}{9 a^{3}}\right.\right. \\
& +\frac{k^{2}}{H^{2}}\left(\frac{1}{5}+\frac{\ln ^{2}(a)}{a^{2}}-\frac{8 \ln (a)}{3 a^{2}}+\frac{16}{9 a^{2}}-\frac{2}{a^{3}}-\frac{4 a^{-5}}{45}\right)-\frac{k^{4}}{H^{4}}\left(\frac{3}{140}-\frac{a^{-2}}{5}+\frac{\ln (a)}{a^{4}}-\frac{11 a^{-4}}{18}+\frac{4 a^{-5}}{5}-\frac{4 a^{-7}}{105}\right)
\end{aligned}
$$




$$
\begin{aligned}
& \left.+\mathcal{O}\left(\frac{k^{6}}{H^{6}}\right)\right]+\frac{\lambda^{2}}{2^{7} 3^{2} \pi^{4}}\left[\frac{7 \ln ^{4}(a)}{3}-\ln ^{3}(a)-\left[\frac{539}{36}-\frac{4 \pi^{2}}{3}-9 \ln \left(\frac{2 \mu}{H}\right)\right] \ln ^{2}(a)+\left[\frac{817}{54}+\frac{5 \pi^{2}}{9}+6 \ln ^{2}\left(\frac{2 \mu}{H}\right)\right.\right. \\
& \left.-16 \ln \left(\frac{2 \mu}{H}\right)-16 \zeta(3)+\sum_{p=2}^{\infty} \sum_{n=1}^{p-1} \frac{24}{n(p-n) p(p+3)}+\left(\frac{17}{18}+\frac{4 \pi^{2}}{9}\right) a^{-3}\right] \ln (a)-\frac{27 a^{-2}}{2}+\mathcal{O}\left(a^{-3}\right) \\
& +\frac{k^{2}}{H^{2}}\left(\frac{2 \ln ^{2}(a)}{5}+\left[\frac{12683}{2250}-\frac{4 \pi^{2}}{15}\right] \ln (a)+\left[\frac{7 \ln ^{2}(a)}{3}-\frac{31 \ln (a)}{3}+9 \ln \left(\frac{2 \mu}{H}\right)+\frac{437}{36}+\frac{4 \pi^{2}}{3}\right] \frac{\ln ^{2}(a)}{a^{2}}\right. \\
& +\left[6 \ln ^{2}\left(\frac{2 \mu}{H}\right)-34 \ln \left(\frac{2 \mu}{H}\right)-16 \zeta(3)-\frac{56}{3}-\frac{19 \pi^{2}}{9}+\sum_{p=2}^{\infty} \sum_{n=1}^{p-1} \frac{24}{n(p-n) p(p+3)}+\mathcal{O}\left(a^{-1}\right)\right] \frac{\ln (a)}{a^{2}}+\frac{\mathcal{C}_{2}}{3} \\
& \left.+\mathcal{O}\left(a^{-2}\right)\right)-\frac{k^{4}}{H^{4}}\left(\frac{3 \ln ^{2}(a)}{70}+\left[\frac{533467}{514500}-\frac{2 \pi^{2}}{35}\right] \ln (a)-\left[\frac{2}{5}+\mathcal{O}\left(\frac{\ln (a)}{a^{2}}\right)\right] \frac{\ln ^{2}(a)}{a^{2}}\right. \\
& \left.\left.\left.+\left[\frac{4 \pi^{2}}{15}-\frac{10883}{2250}+\mathcal{O}\left(a^{-1}\right)\right] \frac{\ln (a)}{a^{2}}-\frac{1}{50}+\frac{\mathcal{C}_{4}}{3}+\mathcal{O}\left(a^{-2}\right)\right)+\mathcal{O}\left(\frac{k^{6}}{H^{6}}\right)\right]\right\} .
\end{aligned}
$$

Thus, the quantum corrections induce $k$-dependence to tree-order power spectrum (94) in even powers of $k / H$, in both loop orders. To fix the $k$-dependent constant terms in the spectrum unambiguously, the order $-\lambda$ and $-\lambda^{2}$ initial state corrections must be worked out in the mode function stage of the calculation, as discussed in Secs. IV A-B. We read off from Eq. (97) that, at one-loop order, the leading term is quadratic in time, whereas the $k$-dependent terms are constants. At two-loop order, on the other hand, the leading term is quartic in time, whereas the $k$-dependent terms, in leading order, are quadratic in time.

The spectral index $n$ measures the variation of the power spectrum of fluctuations in a field with scale. We may quantify the spectral index $n_{\varphi}$ of our scalar field $\varphi(x)$ the same way as for the graviton,

$$
n_{\varphi}(t, k)=\frac{d \ln \left(\Delta_{\varphi}^{2}(t, k)\right)}{d \ln (k)} .
$$

The prediction we would like to make for a cosmological observable like the spectral index $n$ of fluctuations in a field is for a realistic evolution in which inflation ends - unlike the pure de Sitter background of our model where inflation lasts forever. If we had the exact scenario we could compute whatever a full mode function imprints itself upon an observable at the time of recombination, a long — but finite - time after the end of inflation. Unfortunately, we can't get what we really want for our spectator scalar $\varphi(x)$ for many reasons:

(i) We don't know how the scalar — which is not measured today - communicates with observables like the metric fluctuation, which is measured;

(ii) We don't know the actual expansion history in which the scalar evolves;

(iii) We don't have even the tree-order solution for our mode function in an arbitrary 
expansion history, etc. One common technique to bypass such unknown details is the WKB method. One treats the tree-order mode function as evolving in an arbitrary background in this method, and finds that it approaches a constant for each mode after horizon crossing. (The fluctuation is frozen to a constant value outside horizon.) That constant can be expressed, in terms of the value of the mode function at time $t=t_{k}$ of first horizon crossing of the fluctuation with mode $k$. This is an approximate way of getting the constant that the mode function, and hence an observable defined via the mode function, approaches at tree-order.

Now, let's consider our quantum loop corrections some of which are time dependent due to the infrared logarithms. They really do not approach constants, although they only grow very slowly. If the spacetime background were pure de Sitter, they would continue to grow forever. The background, however, cannot really be pure de Sitter, because inflation had to end. To estimate how big the $\ln (a)$ got, we define the number $\mathcal{N}$ of e-foldings after first horizon crossing until the end of inflation. Of course $\mathcal{N}$ differs for each mode, but not much for the window of observable modes. It's about 50, and this is a reasonable estimate for the value of $\ln (a)$.

At time $t_{k}$ the fluctuation with physical wave number $k_{\text {phys }}(t)=k / a(t)$ crosses the horizon for the first time, hence $k_{\text {phys }}\left(t_{k}\right)=H\left(t_{k}\right)$. Because the expansion rate $H$ is constant during de Sitter inflation, this mode has comoving wave number

$$
k=H a\left(t_{k}\right)=H e^{H t_{k}}
$$

Using Eqs. (97)-(99) we find the spectral index for the mode $k$ at the time of first horizon crossing, up to $\mathcal{O}\left(\lambda^{3}\right)$, as

$$
\begin{aligned}
& n_{\varphi}\left(t_{k}, k\right)=\frac{1}{\Delta_{\varphi}^{2}\left(t_{k}, k\right)}\left[k \frac{\partial}{\partial k}+a\left(t_{k}\right) \frac{\partial}{\partial a\left(t_{k}\right)}\right] \Delta_{\varphi}^{2}\left(t_{k}, k\right) \\
& =-\frac{\lambda}{12 \pi^{2}}\left[\ln (a)-\frac{1}{3}+\frac{a^{-3}}{3}+\frac{k^{2}}{H^{2}}\left(\frac{1}{5}-a^{-2}+a^{-3}+\mathcal{O}\left(a^{-5}\right)\right)-\frac{k^{4}}{H^{4}}\left(\frac{3}{70}+\frac{\ln (a)}{a^{4}}+\mathcal{O}\left(a^{-4}\right)\right)+\mathcal{O}\left(\frac{k^{6}}{H^{6}}\right)\right] \\
& +\frac{\lambda^{2}}{216 \pi^{4}}\left[\ln ^{3}(a)+\frac{3 \ln ^{2}(a)}{16}-\left(\frac{185}{32}-\frac{\pi^{2}}{2}-\frac{27}{8} \ln \left(\frac{2 \mu}{H}\right)\right) \ln (a)+\frac{817}{288}+\frac{5 \pi^{2}}{48}+\frac{9}{8} \ln ^{2}\left(\frac{2 \mu}{H}\right)-3 \ln \left(\frac{2 \mu}{H}\right)\right. \\
& -3 \zeta(3)+\frac{9}{2} \sum_{p=2}^{\infty} \sum_{n=1}^{p-1} \frac{1}{n(p-n) p(p+3)}+\frac{81}{16 a^{2}}-\frac{\ln ^{2}(a)}{4 a^{3}}-\left(\frac{19}{96}+\frac{\pi^{2}}{4}\right) \frac{\ln (a)}{a^{3}}+\mathcal{O}\left(a^{-3}\right) \\
& +\frac{k^{2}}{H^{2}}\left(\left(\frac{13283}{6000}-\frac{\pi^{2}}{10}\right) \ln (a)+\frac{13283}{12000}-\frac{\pi^{2}}{20}+\frac{\mathcal{C}_{2}}{8}-\frac{3 \ln ^{3}(a)}{2 a^{2}}-\frac{3 \ln ^{2}(a)}{2 a^{2}}+\frac{15 \ln (a)}{2 a^{2}}+\mathcal{O}\left(a^{-2}\right)\right)
\end{aligned}
$$


$\left.-\frac{k^{4}}{H^{4}}\left(\left(\frac{548167}{686000}-\frac{3 \pi^{2}}{70}\right) \ln (a)+\frac{589327}{2744000}-\frac{3 \pi^{2}}{280}+\frac{\mathcal{C}_{4}}{4}+\frac{3 \ln ^{2}(a)}{10 a^{2}}+\frac{\ln (a)}{10 a^{2}}+\mathcal{O}\left(a^{-2}\right)\right)+\mathcal{O}\left(\frac{k^{6}}{H^{6}}\right)\right]$

where the terms that depend on the scale factor $a$ in Eq. (101) must be evaluated at $t=t_{k}$. To get a reasonable estimate of $n_{\varphi}(t, k)$ after the end of inflation, $\ln (a)$ may be taken as the number of e-foldings $\mathcal{N} \sim 50$. We infer from Eq. (101) that the dominant one-loop quantum effect red tilts the spectrum — although at two-loop order the effect contributes as a blue tilt. Therefore, the amplitudes of fluctuations grow slightly towards the larger scales. How

much the spectral index $n_{\varphi}$ changes as the scale varies is measured by the running of the index

$$
\alpha_{\varphi}(t, k)=\frac{d n_{\varphi}(t, k)}{d \ln (k)} .
$$

At the time of first horizon crossing

$$
\begin{gathered}
\alpha_{\varphi}\left(t_{k}, k\right)=-\frac{\lambda}{12 \pi^{2}}\left[1-a^{-3}+\frac{k^{2}}{H^{2}}\left(\frac{2}{5}-a^{-3}+\frac{3}{5 a^{5}}\right)-\frac{k^{4}}{H^{4}}\left(\frac{6}{35}+a^{-4}-\frac{3}{5 a^{5}}-\frac{4}{7 a^{7}}\right)+\mathcal{O}\left(\frac{k^{6}}{H^{6}}\right)\right] \\
+\frac{\lambda^{2}}{72 \pi^{4}}\left[\ln ^{2}(a)+\frac{\ln (a)}{8}-\frac{185}{96}+\frac{\pi^{2}}{6}+\frac{9}{8} \ln \left(\frac{2 \mu}{H}\right)-\frac{27}{8 a^{2}}+\frac{\ln ^{2}(a)}{4 a^{3}}+\left(\frac{1}{32}+\frac{\pi^{2}}{4}\right) \frac{\ln (a)}{a^{3}}+\mathcal{O}\left(a^{-3}\right)\right. \\
+\frac{k^{2}}{H^{2}}\left(\left(\frac{13283}{9000}-\frac{\pi^{2}}{15}\right)(\ln (a)+1)+\frac{\mathcal{C}_{2}}{12}-\frac{3 \ln ^{2}(a)}{2 a^{2}}-\frac{\ln (a)}{a^{2}}+\mathcal{O}\left(a^{-2}\right)\right)-\frac{k^{4}}{H^{4}}\left(\left(\frac{548167}{514500}-\frac{2 \pi^{2}}{35}\right) \ln (a)\right. \\
\left.\left.+\frac{568747}{1029000}-\frac{\pi^{2}}{35}+\frac{\mathcal{C}_{4}}{3}+\frac{\ln ^{2}(a)}{5 a^{2}}+\frac{4 \ln (a)}{15 a^{2}}+\mathcal{O}\left(a^{-2}\right)\right)+\mathcal{O}\left(\frac{k^{6}}{H^{6}}\right)\right]+\mathcal{O}\left(\lambda^{3}\right) .
\end{gathered}
$$

The terms that depend on $a$ in Eq. (103) are evaluated at $t=t_{k}$. At one-loop order the leading terms of $\alpha_{\varphi}$ are constants at zeroth, quadratic and quartic order in $k / H$. After the first horizon crossing, inflationary expansion enhances this result only at two-loop order. Thus, the running of the spectral index towards larger scales is indeed slight.

\section{CONCLUSIONS}

We have solved the one- and two-loop corrected, linearized effective field equation for a massless, minimally coupled scalar endowed with a quartic self-interaction in locally de Sitter background for a state released in Bunch-Davies vacuum at time $t=0$. The solution is the quantum corrected mode function of the scalar at one- and two-loop orders. The quantum corrections can be expanded in powers of $k / H$ and are proportional to $u(\infty, k)$; the late-time limit of the tree-order mode function. See Eqs. (61)-(62) and (89). Note that, 
the shifts linear in $k / H$ are absent in both loop corrections. At one-loop order, the quantum shift that is zero order in $k / H$ grows proportional to $(H t)^{2}$ whereas the second and higher order shifts in $k / H$ are constants, in leading order. At two-loop order, on the other hand, the quantum shift that is zero order in $k / H$ grows proportional to $(H t)^{4}$, whereas the second and higher order shifts in $k / H$ grow proportional to $(H t)^{2}$, in leading order.

We have also computed the scalar's power spectrum $\Delta_{\varphi}^{2}(t, k)$. Quantum corrections induce scale dependent shifts in even powers of $k / H$ to the scale-free tree-order late-time spectrum; see Eq. (97). At one loop order, the quantum shift that is zero order in $k / H$ grows proportional to $(H t)^{2}$ whereas the shifts quadratic and quartic in $k / H$ are constants, in leading order. At two-loop order, the quantum shift that is zero order in $k / H$ grows proportional to $(H t)^{4}$, whereas the shifts quadratic and quartic in $k / H$ grows proportional to $(H t)^{2}$, in leading order. The spectral index $n_{\varphi}(t, k)$ vanishes at tree-order; see Eq. (101). It is purely quantum mechanical in origin. At one-loop order, the quantum shift that is zero order in $k / H$ grows proportional to $H t$ whereas the shifts quadratic and quartic in $k / H$ are constants, in leading order. At two-loop order, on the other hand, the quantum shift that is zero order in $k / H$ grows proportional to $(H t)^{3}$, whereas the shifts quadratic and quartic in $k / H$ grows proportional to $H t$, in leading order. The running of the spectral index $\alpha_{\varphi}(t, k)$ is given in Eq. (103). We conclude that the quantum effects red-tilts the spectrum slightly. Hence, the amplitudes of fluctuations grow towards the larger scales.

Because we cannot even measure the amplitude of our spectator scalar, currently there isn't any data on either its spectral index or its tilt. Can, in principle, the quantum shifts we calculated in the spectrum be detected, assuming we can measure the tree-order power spectrum for the scalar we consider? The answer to the question depends upon how sensitively we can measure the spectrum and what the value of the self-coupling $\lambda$ is. One also needs to be able to distinguish between any loop correction and a change in the inflaton potential.

A phenomenologically more interesting application of the computation presented in this paper would be tying it to Higgs inflation [20]. For Higgs inflation one needs the scalar background not to be $\varphi=0$. One also needs a strong conformal coupling — so the scalar would not any longer be even approximately minimally coupled. 


\section{Appendix A: Evaluating the functional $\xi(\alpha)$}

In Sec. IV B, while evaluating $\mathcal{I}_{1}(\eta, k)$, we defined the functional $\xi(\alpha)$ in Eq. (81) as

$$
\xi(\alpha) \equiv \int_{0}^{1} d z z \sin (\alpha z) \ln \left(1-z^{2}\right)=\int_{0}^{1} d z z \sin (\alpha z)[\ln (1-z)+\ln (1+z)] .
$$

In this appendix we evaluate the $\xi(\alpha)$. The first step is to make the change of variable $y=1-z$ for the integral involving $\ln (1-z)$ and $y=1+z$ for the other integral involving $\ln (1+z)$. Then, we find

$$
\xi(\alpha)=\int_{0}^{2} d y(1-y) \ln (y) \sin (\alpha(1-y))=-\frac{d}{d \alpha} \int_{0}^{2} d y \ln (y) \cos (\alpha-\alpha y) .
$$

Expanding out the cosine term and changing the variable from $y$ to $x=\alpha y$, we obtain

$$
\xi(\alpha)=-\frac{d}{d \alpha}\left\{\frac{1}{\alpha}\left[\cos (\alpha) \int_{0}^{2 \alpha} d x \ln (x) \cos (x)+\sin (\alpha) \int_{0}^{2 \alpha} d x \ln (x) \sin (x)-2 \ln (\alpha) \sin (\alpha)\right]\right\}
$$

Finally, recognizing the sine integral $\operatorname{si}(\alpha)$ and cosine integral $\operatorname{ci}(\alpha)$ in the form

$$
\begin{aligned}
& \int_{0}^{2 \alpha} d x \ln (x) \cos (x)=\ln (2 \alpha) \sin (2 \alpha)-\frac{\pi}{2}-\operatorname{si}(2 \alpha), \\
& \int_{0}^{2 \alpha} d x \ln (x) \sin (x)=-\ln (2 \alpha) \cos (2 \alpha)-\gamma+\operatorname{ci}(2 \alpha),
\end{aligned}
$$

where

$$
\begin{aligned}
& \operatorname{si}(\alpha) \equiv-\int_{\alpha}^{\infty} d t \frac{\sin (t)}{t}=-\frac{\pi}{2}+\int_{0}^{\alpha} d t \frac{\sin (t)}{t} \\
& \operatorname{ci}(\alpha) \equiv-\int_{\alpha}^{\infty} d t \frac{\cos (t)}{t}=\gamma+\ln (\alpha)+\int_{0}^{\alpha} d t \frac{\cos (t)-1}{t}
\end{aligned}
$$

with $\gamma \approx .577$ being the Euler's constant, and using the identities

$$
\frac{d}{d \alpha} \operatorname{si}(\alpha)=\frac{\sin (\alpha)}{\alpha}, \quad \frac{d}{d \alpha} \operatorname{ci}(\alpha)=\frac{\cos (\alpha)}{\alpha},
$$

we find

$$
\begin{aligned}
\xi(\alpha)=\frac{2}{\alpha^{2}} \sin (\alpha) & -\frac{1}{\alpha^{2}}[\cos (\alpha)+\alpha \sin (\alpha)]\left[\operatorname{si}(2 \alpha)+\frac{\pi}{2}\right] \\
+ & \frac{1}{\alpha^{2}}[\sin (\alpha)-\alpha \cos (\alpha)]\left[\operatorname{ci}(2 \alpha)-\ln \left(\frac{\alpha}{2}\right)-\gamma\right]
\end{aligned}
$$

The functional $\xi(\alpha)$ also appears in the evaluations of the integrals $I_{2}(\eta, k), I_{3}(\eta, k), I_{4}(\eta, k)$ and $I_{5}(\eta, k)$; see Eqs. (B1), (B7), (B15) and (B19), respectively. Note that the following 
expansions of the $\operatorname{si}(\alpha)$ and $\operatorname{ci}(\alpha)$ are useful through out the paper

$$
\begin{aligned}
\operatorname{si}(\alpha)+\frac{\pi}{2} & =\sum_{n=1}^{\infty} \frac{(-1)^{n+1} \alpha^{2 n-1}}{(2 n-1)(2 n-1) !} \\
\operatorname{ci}(\alpha)-\ln (\alpha)-\gamma & =\sum_{n=1}^{\infty} \frac{(-1)^{n} \alpha^{2 n}}{2 n(2 n) !} .
\end{aligned}
$$

\section{Appendix B: Integrating $\mathcal{I}_{n}(\eta, k)$ for $2 \leq n \leq 6$}

In Sec. IV B, we obtained the integro-differential equation (65) for the two-loop correction $\Phi_{2}(\eta, k)$ to the scalar mode function. In the source term of the equation we defined integrals $\mathcal{I}_{n}(\eta, k)$ (Eq. (68)) and evaluated $\mathcal{I}_{1}(\eta, k)$ through equations (71)-(86). The evaluation of $\mathcal{I}_{2}(\eta, k)$ is similar to the one for $\mathcal{I}_{1}(\eta, k)$. Defining $\beta \equiv \ln \left(\frac{H e^{\frac{3}{4}}}{2 \mu}\right)$, we write

$$
\mathcal{I}_{2}(\eta, k)=\frac{i H^{2} \beta}{2^{9} \pi^{6} a^{2}} \int d^{4} x^{\prime} a^{\prime 2}\left\{\partial^{2}\left[\frac{\ln \left(\mu^{2} \Delta x_{++}^{2}\right)}{\Delta x_{++}^{2}}-\frac{\ln \left(\mu^{2} \Delta x_{+-}^{2}\right)}{\Delta x_{+-}^{2}}\right]\right\} u\left(\eta^{\prime}, k\right) e^{-i \vec{k} \cdot\left(\vec{x}-\vec{x}^{\prime}\right)} .
$$

Following the steps from Eq. (72) to Eq. (80) we obtain

$$
\mathcal{I}_{2}(\eta, k)=\frac{-H^{2} \beta}{2^{7} \pi^{4}} \frac{\left(\partial_{0}^{2}+k^{2}\right)^{2}}{a^{2} k^{3}} \int_{\eta_{i}}^{\eta} d \eta^{\prime} a^{\prime 2} u\left(\eta^{\prime}, k\right)\left\{\left[\ln (\mu \Delta \eta)-\frac{1}{2}\right][\sin (\alpha)-\alpha \cos (\alpha)]+\frac{\alpha^{2}}{2} \xi(\alpha)\right\}
$$

Applying the derivatives using Eq. (83) yields

$$
\begin{array}{r}
\mathcal{I}_{2}(\eta, k)=\frac{-H^{2} \beta}{2^{7} \pi^{4}} \frac{\left(\partial_{0}^{2}+k^{2}\right)}{a^{2} k} \int_{\eta_{i}}^{\eta} d \eta^{\prime} a^{\prime 2} u\left(\eta^{\prime}, k\right)\{[\operatorname{ci}(2 \alpha)-\ln (2 \alpha)-\gamma+2 \ln (2 \mu \Delta \eta)] \sin (\alpha) \\
\left.-\left[\operatorname{si}(2 \alpha)+\frac{\pi}{2}\right] \cos (\alpha)\right\} \\
=\frac{-H^{2} \beta}{2^{6} \pi^{4}} a^{-2}\left\{k \int_{\eta_{i}}^{\eta} d \eta^{\prime} a^{\prime 2} u\left(\eta^{\prime}, k\right) \ln (2 \mu \Delta \eta) \sin (\alpha)+\partial_{0} \int_{\eta_{i}}^{\eta} d \eta^{\prime} a^{\prime 2} u\left(\eta^{\prime}, k\right) \ln (2 \mu \Delta \eta) \cos (\alpha)\right\} .
\end{array}
$$

We expand the logarithms using Eq. (85) and evaluate the integrals. Taking the necessary derivative and expressing the final result in powers of $k / a$ we finally find

$$
\begin{aligned}
& \mathcal{I}_{2}(\eta, k)=\frac{\beta}{2^{6} \pi^{4}} \frac{H}{\sqrt{2 k^{3}}}\left\{H^{2}\left[\ln (a)-\ln \left(\frac{2 \mu}{H e}\right)+\sum_{n=1}^{\infty} \frac{a^{-(n+1)}}{n+1}\right]\right. \\
&-\frac{k^{2}}{a^{2}}\left[\frac{1}{2} \ln \left(\frac{2 \mu}{H a}\right)+\frac{1}{2}-a^{-1}-\frac{1}{2} \sum_{n=1}^{\infty} \frac{a^{-(n+1)}}{n+1}\right]-\frac{i k^{3}}{H a^{3}}\left[\frac{a}{3}+\frac{1}{3} \ln \left(\frac{2 \mu}{H e}\right)-\frac{a^{-1}}{3}-\frac{1}{3} \sum_{n=1}^{\infty} \frac{a^{-(n+1)}}{n+1}\right] \\
&\left.+\frac{k^{4}}{H^{2} a^{4}}\left[\frac{a^{2}}{6}-\frac{1}{8} \ln (a)+\frac{1}{8} \ln \left(\frac{2 \mu}{H e}\right)-\frac{a^{-1}}{6}-\frac{1}{8} \sum_{n=1}^{\infty} \frac{a^{-(n+1)}}{n+1}\right]+\mathcal{O}\left(\frac{i k^{5}}{H^{3}}\right)\right\} .(\mathrm{B} 3)
\end{aligned}
$$


To evaluate the third integral

$$
\mathcal{I}_{3}(\eta, k) \equiv \frac{i H^{2}}{2^{11} \pi^{6} a^{2}} \int d^{4} x^{\prime} a^{\prime 2}\left\{\partial^{2}\left[\frac{\ln ^{2}\left(\mu^{2} \Delta x_{++}^{2}\right)}{\Delta x_{++}^{2}}-\frac{\ln ^{2}\left(\mu^{2} \Delta x_{+-}^{2}\right)}{\Delta x_{+-}^{2}}\right]\right\} u\left(\eta^{\prime}, k\right) e^{-i \vec{k} \cdot\left(\vec{x}-\vec{x}^{\prime}\right)},
$$

in the source term (70) of the integro-differential equation (69), we first use the identity

$$
\frac{\ln ^{2}\left(\mu^{2} \Delta x^{2}\right)}{\Delta x^{2}}=\frac{\partial^{2}}{12}\left[\ln ^{3}\left(\mu^{2} \Delta x^{2}\right)-3 \ln ^{2}\left(\mu^{2} \Delta x^{2}\right)+6 \ln \left(\mu^{2} \Delta x^{2}\right)\right] .
$$

Then, the usual cancelation between the ++ and +- terms outside light-cone via Eq. (73) converts the integral to the form

$$
\begin{aligned}
\mathcal{I}_{3}(\eta, k)=\frac{-H^{2}}{2^{12} \pi^{5}} e^{-i \vec{k} \cdot \vec{x}} \frac{\partial^{4}}{a^{2}} \int_{\eta_{i}}^{\eta} d \eta^{\prime} a^{\prime 2} u\left(\eta^{\prime}, k\right) \int d^{3} x^{\prime} e^{i \vec{k} \cdot \vec{x}^{\prime}} \Theta(\Delta \eta-r) \\
\quad \times\left\{\ln ^{2}\left(\mu^{2}\left(\Delta \eta^{2}-r^{2}\right)\right)-2 \ln \left(\mu^{2}\left(\Delta \eta^{2}-r^{2}\right)\right)-\frac{\pi^{2}}{3}+2\right\} .
\end{aligned}
$$

Evaluating the angular integrations using Eq. (76), passing the spatial phase factor through the derivative using $\partial^{4} e^{i \vec{k} \cdot \vec{x}}=e^{i \vec{k} \cdot \vec{x}}\left(\partial_{0}^{2}+k^{2}\right)^{2}$ and making the usual change of variable $r \equiv$ $\Delta \eta z \equiv \frac{\alpha}{k} z$ yields

$$
\begin{aligned}
\mathcal{I}_{3}(\eta, k)=\frac{-H^{2}}{2^{8} \pi^{4}} \frac{\left(\partial_{0}^{2}+k^{2}\right)^{2}}{a^{2} k^{3}} \int_{\eta_{i}}^{\eta} d \eta^{\prime} a^{\prime 2} u\left(\eta^{\prime}, k\right)\left\{\left[\ln ^{2}(\mu \Delta \eta)-\ln (\mu \Delta \eta)-\ln ^{2}(2)-\frac{\pi^{2}}{12}+\frac{1}{2}\right]\right. \\
\left.\times[\sin (\alpha)-\alpha \cos (\alpha)]+\left[\ln (2 \mu \Delta \eta)-\frac{1}{2}\right] \alpha^{2} \xi(\alpha)+\frac{\alpha^{2}}{4} \mathcal{W}(\alpha)\right\}
\end{aligned}
$$

where we define a new functional

$$
\begin{aligned}
\mathcal{W}(\alpha) & =\int_{0}^{1} d z z \sin (\alpha z) \ln ^{2}\left(\frac{1-z^{2}}{4}\right) \\
& =\sum_{n=0}^{\infty} \frac{(-1)^{n} \alpha^{2 n+1}}{(2 n+1) !} \int_{0}^{1} d z z^{2 n+2} \ln ^{2}\left(\frac{1-z^{2}}{4}\right) \\
& =\left(\frac{104}{27}-\frac{\pi^{2}}{9}\right) \alpha-\left(\frac{1576}{3375}-\frac{\pi^{2}}{90}\right) \alpha^{3}+\left(\frac{21946}{1157625}-\frac{\pi^{2}}{2520}\right) \alpha^{5}+O\left(\alpha^{7}\right) .
\end{aligned}
$$

See Appendix D for the evaluation of the $\mathcal{W}(\alpha)$. Applying $\frac{\partial_{0}^{2}+k^{2}}{k^{2}}$ in Eq. (B7) yields

$$
\begin{aligned}
\mathcal{I}_{3}(\eta, k) & =\frac{-H^{2}}{2^{8} \pi^{4}} \frac{\left(\partial_{0}^{2}+k^{2}\right)}{a^{2} k} \int_{\eta_{i}}^{\eta} d \eta^{\prime} a^{\prime 2} u\left(\eta^{\prime}, k\right)\left\{\operatorname { s i n } ( \alpha ) \left\{\frac{\operatorname{si}(2 \alpha)+\frac{\pi}{2}}{\alpha}+2 \ln ^{2}(2 \mu \Delta \eta)-\frac{\pi^{2}}{6}\right.\right. \\
& \left.+[\operatorname{ci}(2 \alpha)-\ln (2 \alpha)-\gamma]\left[2 \ln (2 \mu \Delta \eta)+1-\frac{1}{\alpha^{2}}\right]-\frac{4}{\alpha^{2}}\right\}+\cos (\alpha)\left\{\frac{\operatorname{ci}(2 \alpha)-\ln (2 \alpha)-\gamma+2}{\alpha}\right. \\
& \left.\left.-\left[\operatorname{si}(2 \alpha)+\frac{\pi}{2}\right]\left[2 \ln (2 \mu \Delta \eta)+1-\frac{1}{\alpha^{2}}\right]\right\}+\frac{\alpha^{2}}{4} \mathcal{W}(\alpha)+\frac{\partial_{0}^{2}}{k^{2}}\left[\frac{\alpha^{2}}{4} \mathcal{W}(\alpha)\right]\right\}
\end{aligned}
$$


Then, we use Eqs. (85), (A9)-(A10) in Eq. (B11), evaluate the integrals over the past lightcone and take the remaining derivatives. Expressing the result in powers of $k / a$, we find

$$
\begin{aligned}
& \mathcal{I}_{3}(\eta, k)=\frac{-1}{2^{8} \pi^{4}} \frac{H}{\sqrt{2 k^{3}}}\left\{H ^ { 2 } \left[2 \ln ^{2}\left(\frac{2 \mu}{H a}\right)-4 \ln \left(\frac{2 \mu}{H a}\right)+\frac{\pi^{2}}{3}+4 \sum_{n=1}^{\infty} \frac{n a^{-(n+1)}}{(n+1)^{2}}-4 \ln \left(\frac{2 \mu}{H}\right) \sum_{n=1}^{\infty} \frac{a^{-(n+1)}}{n+1}\right.\right. \\
& +2 \sum_{p=2}^{\infty} \sum_{n=1}^{p-1} \frac{a^{-(p+1)}}{n(p-n)}[p-(p-1) a]+\frac{k^{2}}{a^{2}}\left[\ln ^{2}\left(\frac{2 \mu}{H a}\right)+2 \ln \left(\frac{2 \mu}{H}\right)-\frac{\pi^{2}}{18}-\frac{16}{9}+a^{-1}\left[2-4 \ln \left(\frac{2 \mu}{H}\right)\right]\right. \\
& +a^{-2}\left[\frac{31}{6}-\ln \left(\frac{2 \mu}{H}\right)\right]-a^{-3}\left[\frac{32}{27}+\frac{2}{3} \ln \left(\frac{2 \mu}{H}\right)\right]+\frac{2}{3} \sum_{n=3}^{\infty} \frac{\left(3 n^{2}-2\right) a^{-(n+1)}}{n(n+1)^{2}}-2 \ln \left(\frac{2 \mu}{H}\right) \sum_{n=3}^{\infty} \frac{a^{-(n+1)}}{n+1} \\
& \left.+\sum_{p=3}^{\infty} \sum_{n=1}^{p-1} \frac{1}{n(p-n)}\left\{\frac{4}{(p-2)(p+1)}-a^{-(p+1)} \frac{p}{3}\left(\frac{p+5}{p+1}-a \frac{3(p+3)}{p}+a^{3} \frac{2(p-1)}{p-2}\right)\right\}\right] \\
& +\frac{i k^{3}}{H a^{3}}\left[\frac{4}{3} \ln \left(\frac{2 \mu}{H e}\right) a-\frac{440 \ln (a)}{63}+\frac{\pi^{2}}{9}+\frac{92}{189}+\frac{2}{3} \ln { }^{2}\left(\frac{2 \mu}{H}\right)-\frac{4}{3} \ln \left(\frac{2 \mu}{H}\right)+8 a^{-1}+\frac{4}{3} \sum_{n=0}^{\infty} \frac{n a^{-(n+1)}}{(n+1)^{2}}\right. \\
& \left.-\frac{4}{3} \ln \left(\frac{2 \mu}{H}\right) \sum_{n=0}^{\infty} \frac{a^{-(n+1)}}{(n+1)}+\sum_{p=4 n=1}^{\infty} \sum_{n-1}^{p(p-n)}\left\{\frac{1}{(p-3)(p-2)}+\frac{2}{3} a^{-(p-2)} p\left(\frac{p+1}{p-2}-a \frac{(p-1)}{p-3}\right)\right\}\right] \\
& +\frac{k^{4}}{H^{2} a^{4}}\left[a^{2}\left[\frac{13}{18}-\frac{2}{3} \ln \left(\frac{2 \mu}{H}\right)\right]+a+\frac{1177}{900}+\frac{\pi^{2}}{40}+\frac{1}{2} \ln \left(\frac{2 \mu}{H a}\right)-\frac{1}{4} \ln { }^{2}\left(\frac{2 \mu}{H a}\right)+a^{-1}\left[\frac{2}{3} \ln \left(\frac{2 \mu}{H}\right)-\frac{20}{3}\right]\right. \\
& +a^{-2}\left[\frac{407}{120}+\frac{1}{4} \ln \left(\frac{2 \mu}{H}\right)\right]+a^{-3}\left[\frac{1}{6} \ln \left(\frac{2 \mu}{H}\right)-\frac{187}{225}\right]-\frac{497}{720} a^{-4}+\frac{143}{900} a^{-5} \\
& -\frac{1}{30} \sum_{n=3}^{\infty} \frac{\left(15 n^{4}+75 n^{3}+110 n^{2}+20 n-24\right) a^{-(n+1)}}{n(n+1)^{2}(n+2)(n+3)}+\frac{1}{2} \ln \left(\frac{2 \mu}{H}\right) \sum_{n=3}^{\infty} \frac{a^{-(n+1)}}{n+1} \\
& -\sum_{p=5}^{\infty} \sum_{n=1}^{p-1} \frac{1}{n(p-n)}\left\{\frac{2\left(p^{2}+p+4\right)}{(p-4)(p-3)(p-2)(p+1)}\right. \\
& \left.\left.-(p+1) p\left(\frac{p+9}{60(p+1)}-a \frac{(p+7)}{12 p}+a^{3} \frac{(p+3)}{3(p-2)}-a^{4} \frac{2(p+1)}{3(p-3)}+a^{5} \frac{2(p-1)}{5(p-4)}\right)\right\}+\mathcal{O}\left(\frac{i k^{5}}{H^{3}}\right)\right\} \\
& (\mathrm{B} 12)
\end{aligned}
$$

The fourth integral in source term (70)

$$
\mathcal{I}_{4}(\eta, k)=\frac{i H^{4}}{2^{9} \pi^{6}} \frac{1}{a} \int d^{4} x^{\prime} a^{\prime 3}\left\{\frac{\ln ^{2}\left(\frac{\sqrt{e} H^{2}}{4} \Delta x_{++}^{2}\right)}{\Delta x_{++}^{2}}-\frac{\ln ^{2}\left(\frac{\sqrt{e} H^{2}}{4} \Delta x_{+-}^{2}\right)}{\Delta x_{+-}^{2}}\right\} u\left(\eta^{\prime}, k\right) e^{-i \vec{k} \cdot\left(\vec{x}-\vec{x}^{\prime}\right)},
$$

is evaluated similarly to the third one. We use Eq. (B5) in Eq. (B13) and obtain

$$
\begin{aligned}
\mathcal{I}_{4}(\eta, k)=\frac{-H^{4}}{2^{10} \pi^{5}} e^{-i \vec{k} \cdot \vec{x}} \frac{\partial^{2}}{a} \int_{\eta_{i}}^{\eta} d \eta^{\prime} a^{\prime 3} u\left(\eta^{\prime}, k\right) \int d^{3} x^{\prime} e^{i \vec{k} \cdot \vec{x}^{\prime}} \Theta(\Delta \eta-r) \\
\times \\
\left.\times \ln ^{2}\left(\frac{H^{2}\left(\Delta \eta^{2}-r^{2}\right)}{4}\right)-\ln \left(\frac{H^{2}\left(\Delta \eta^{2}-r^{2}\right)}{4}\right)+\frac{5}{4}-\frac{\pi^{2}}{3}\right] .
\end{aligned}
$$

Evaluating the angular integrations using Eq. (76), passing the spatial phase factor through the derivative using $\partial^{2} e^{i \vec{k} \cdot \vec{x}}=-e^{i \vec{k} \cdot \vec{x}}\left(\partial_{0}^{2}+k^{2}\right)$ and then making the change of variable $r \equiv \frac{\alpha}{k} z$ 
reduces Eq. (B14) to the form

$$
\begin{aligned}
\mathcal{I}_{4}(\eta, k)=\frac{H^{4}}{2^{6} \pi^{4}} \frac{\left(\partial_{0}^{2}+k^{2}\right)}{a k^{3}} \int_{\eta_{i}}^{\eta} d \eta^{\prime} a^{\prime 3} u\left(\eta^{\prime}, k\right)\left\{\left[\ln ^{2}\left(\frac{H \Delta \eta}{2}\right)-\frac{1}{2} \ln \left(\frac{H \Delta \eta}{2}\right)-\ln ^{2}(2)-\frac{\pi^{2}}{12}+\frac{5}{16}\right]\right. \\
\left.\times[\sin (\alpha)-\alpha \cos (\alpha)]+\left[\ln (H \Delta \eta)-\frac{1}{4}\right] \alpha^{2} \xi(\alpha)+\frac{\alpha^{2}}{4} \mathcal{W}(\alpha)\right\} .
\end{aligned}
$$

When the derivatives are applied to the integral using Eq. (83), $\mathcal{I}_{4}(\eta, k)$ becomes

$$
\begin{array}{r}
\frac{H^{4}}{2^{6} \pi^{4}} \frac{1}{a k} \int_{\eta_{i}}^{\eta} d \eta^{\prime} a^{\prime 3} u\left(\eta^{\prime}, k\right)\left\{\cos (\alpha)\left\{\left[\frac{1}{\alpha^{2}}-\frac{3}{2}-2 \ln (H \Delta \eta)\right]\left[\operatorname{si}(2 \alpha)+\frac{\pi}{2}\right]+\frac{\operatorname{ci}(2 \alpha)-\ln (2 \alpha)-\gamma+2}{\alpha}\right\}\right. \\
+\sin (\alpha)\left\{\frac{\operatorname{si}(2 \alpha)+\frac{\pi}{2}}{\alpha}-\left[\frac{1}{\alpha^{2}}-\frac{3}{2}-2 \ln (H \Delta \eta)\right][\operatorname{ci}(2 \alpha)-\ln (2 \alpha)-\gamma]-\frac{4}{\alpha^{2}}-\frac{\pi^{2}}{6}+\frac{1}{8}\right. \\
\left.\left.+\ln (H \Delta \eta)+2 \ln ^{2}(H \Delta \eta)\right\}+\frac{\alpha^{2}}{4} \mathcal{W}(\alpha)+\frac{\partial_{0}^{2}}{k^{2}}\left[\frac{\alpha^{2}}{4} \mathcal{W}(\alpha)\right]\right\} .
\end{array}
$$

Then, we use Eqs. (85), (A9)-(A10) in Eq. (B16) and integrate over the past light-cone. In powers of $k / a$ we have

$$
\begin{aligned}
\mathcal{I}_{4} & (\eta, k)=\frac{1}{2^{6} \pi^{4}} \frac{H}{\sqrt{2 k^{3}}}\left\{H ^ { 2 } \left[\ln ^{2}(a)-\frac{\ln (a)}{2}+\frac{\pi^{2}}{6}-\frac{7}{16}+\left(\frac{\pi^{2}}{3}-\frac{33}{8}\right) a^{-1}+\left(\frac{13}{16}-\frac{\pi^{2}}{6}\right) a^{-2}\right.\right. \\
& \left.+\sum_{n=2}^{\infty} \frac{(3 n-1) a^{-(n+1)}}{(n-1) n(n+1)^{2}}+2 \sum_{p=2}^{\infty} \sum_{n=1}^{p-1} \frac{1}{n(p-n)}\left\{\frac{1}{(p+1)(p+2)}-a^{-(p+1)}\left(\frac{1}{p+1}-\frac{a^{-1}}{p+2}\right)\right\}\right] \\
& -\frac{k^{2}}{a^{2}}\left[\frac{\ln ^{2}(a)}{2}+\frac{3 \ln (a)}{4}-\left(\frac{629}{216}-\frac{\pi^{2}}{9}\right) a+\frac{89}{96}+\frac{5 \pi^{2}}{36}-\left(\frac{83}{48}-\frac{\pi^{2}}{6}\right) a^{-1}-\left(\frac{11}{96}+\frac{\pi^{2}}{36}\right) a^{-2}\right. \\
& -\frac{1}{6} \sum_{n=2}^{\infty} \frac{\left(9 n^{2}-21 n-2\right) a^{-(n+1)}}{(n-1) n(n+1)^{2}(n+2)}-\sum_{p=2}^{\infty} \sum_{n=1}^{p-1} \frac{1}{n(p-n)}\left\{\frac{p+3}{(p-1)(p+1)(p+2)}\right. \\
& \left.\left.-a^{-(p-1)}\left(\frac{2}{3(p-1)}-\frac{a^{-2}}{p+1}+\frac{a^{-3}}{3(p+2)}\right)\right\}\right]+\frac{i k^{3}}{H a^{3}}\left[\left(\frac{15}{16}-\frac{\pi^{2}}{18}\right) a^{2}+\left(\frac{\pi^{2}}{9}-\frac{25}{24}\right) a+\left(\frac{\pi^{2}}{18}-\frac{79}{48}\right)\right. \\
& +\frac{11 a^{-1}}{18}+\frac{1}{3} \sum_{n=1}^{\infty} \frac{(3 n-1) a^{-(n+1)}}{(n+1)^{2}(n+2)(n+3)}+\frac{2}{3} \sum_{p=3}^{\infty} \sum_{n=1}^{p-1} \frac{1}{n(p-n)}\left\{\frac{1}{(p-1)(p-2)}\right. \\
& \left.\left.-a^{-(p-2)}\left(\frac{1}{p-2}-\frac{a^{-1}}{p-1}\right)\right\}\right]+\frac{k^{4}}{H^{2} a^{4}}\left[\left(\frac{\pi^{2}}{45}-\frac{9403}{27000}\right) a^{3}+\left(\frac{251}{432}-\frac{\pi^{2}}{18}\right) a^{2}+\left(\frac{\pi^{2}}{18}-\frac{245}{432}\right) a\right. \\
& +\frac{\ln ^{2}(a)}{24}+\frac{13 \ln (a)}{144}+\frac{17 \pi^{2}}{720}+\frac{10157}{86400}-\frac{1}{4} \sum_{p=4}^{\infty} \sum_{n=1}^{p-1} \frac{p^{3}+2 p^{2}+3 p+10}{n(p-n)(p-3)(p-2)(p-1)(p+1)(p+2)} \\
& +\left(\frac{\pi^{2}}{72}-\frac{437}{960}\right) a^{-1}+\left(\frac{839}{5760}-\frac{\pi^{2}}{720}\right) a^{-2}-\frac{107 a^{-3}}{3600}-\frac{2789 a^{-4}}{151200}+\frac{1651 a^{-5}}{756000} \\
& \left.-\frac{1}{120} \sum_{n=5}^{\infty} \frac{\left(45 n^{4}+30 n^{3}+155 n^{2}-470 n-136\right) a^{-(n+1)}}{(n-1) n(n+1)^{2}(n+2)(n+3)(n+4)}\right]+\sum_{p=4}^{\infty} \sum_{n=1}^{p-1} \frac{a^{-(p-3)}}{n(p-n)}\left\{\frac{2}{5(p-3)}\right. \\
& \left.\left.-\frac{2 a^{-1}}{3(p-2)}+\frac{a^{-2}}{3(p-1)}-\frac{a^{-4}}{12(p+1)}+\frac{a^{-5}}{60(p+2)}\right\}+\mathcal{O}\left(\frac{i k^{5}}{H^{3}}\right)\right\} . \quad(\mathrm{B} 17)
\end{aligned}
$$


The fifth integral in the source term involves difference of two cubic logarithms in the integrand,

$$
\mathcal{I}_{5}(\eta, k)=\frac{-i H^{6}}{2^{10} 3 \pi^{6}} \int d^{4} x^{\prime} a^{\prime 4}\left\{\ln ^{3}\left(\frac{\sqrt{e}}{4} H^{2} \Delta x_{++}^{2}\right)-\ln ^{3}\left(\frac{\sqrt{e}}{4} H^{2} \Delta x_{+-}^{2}\right)\right\} u\left(\eta^{\prime}, k\right) e^{-i \vec{k} \cdot\left(\vec{x}-\vec{x}^{\prime}\right)} .
$$

Expanding the difference of two cubes and using Eq. (73) we find

$$
\mathcal{I}_{5}(\eta, k)=\frac{H^{6}}{2^{9} \pi^{5}} e^{-i \vec{k} \cdot \vec{x}} \int_{\eta_{i}}^{\eta} d \eta^{\prime} a^{\prime 4} u\left(\eta^{\prime}, k\right) \int d^{3} x^{\prime} e^{i \vec{k} \cdot \vec{x}^{\prime}} \Theta(\Delta \eta-\Delta x)\left\{\ln ^{2}\left(\frac{\sqrt{e}}{4} H^{2}\left(\Delta \eta^{2}-\Delta x^{2}\right)\right)-\frac{\pi^{2}}{3}\right\} .
$$

Evaluating the angular integrations using Eq. (76) and making the change of variable $r \equiv$ $\Delta \eta z \equiv \frac{\alpha}{k} z$ yields

$$
\begin{aligned}
\mathcal{I}_{5}(\eta, k)=\frac{H^{6}}{2^{5} \pi^{4}} \frac{1}{k^{3}} \int_{\eta_{i}}^{\eta} d \eta^{\prime} a^{\prime 4} u\left(\eta^{\prime}, k\right)\left\{\left[\ln ^{2}\left(\frac{H \Delta \eta}{2}\right)+\frac{1}{2} \ln \left(\frac{H \Delta \eta}{2}\right)-\ln ^{2}(2)+\frac{1}{16}-\frac{\pi^{2}}{12}\right]\right. \\
\left.\times[\sin (\alpha)-\alpha \cos (\alpha)]+\left[\ln (H \Delta \eta)+\frac{1}{4}\right] \alpha^{2} \xi(\alpha)+\frac{\alpha^{2}}{4} \mathcal{W}(\alpha)\right\} .
\end{aligned}
$$

We insert $\xi(\alpha)$ given in Eq. (82) into Eq. (B19) and regroup the terms in the integrand,

$$
\begin{aligned}
& \mathcal{I}_{5}(\eta, k)=\frac{H^{6}}{2^{5} \pi^{4}} \frac{1}{k^{3}} \int_{\eta_{i}}^{\eta} d \eta^{\prime} a^{\prime 4} u\left(\eta^{\prime}, k\right)\left\{\frac{\sin (\alpha)}{2}-\frac{1}{4}\left[\operatorname{si}(2 \alpha)+\frac{\pi}{2}\right][\cos (\alpha)+\alpha \sin (\alpha)]\right. \\
&+\frac{1}{4}\left[\operatorname{ci}(2 \alpha)-\ln (2 \alpha)-\gamma+\frac{1}{4}-\frac{\pi^{2}}{3}+4 \ln ^{2}(H \Delta \eta)\right][\sin (\alpha)-\alpha \cos (\alpha)] \\
&+\ln (H \Delta \eta)\left\{\sin (\alpha)\left(\operatorname{ci}(2 \alpha)-\ln (2 \alpha)-\gamma-\alpha\left[\operatorname{si}(2 \alpha)+\frac{\pi}{2}\right]+\frac{5}{2}\right)\right. \\
&\left.\left.-\cos (\alpha)\left(\operatorname{si}(2 \alpha)+\frac{\pi}{2}+\alpha\left[\operatorname{ci}(2 \alpha)-\ln (2 \alpha)-\gamma+\frac{1}{2}\right]\right)\right\}+\frac{\alpha^{2}}{4} \mathcal{W}(\alpha)\right\} .
\end{aligned}
$$

Using Eqs. (85), (A9)-(A10) in Eq. (B20) and integrating over the past light-cone, we obtain

$$
\begin{aligned}
& \mathcal{I}_{5}(\eta, k)=\frac{1}{2^{5} \pi^{4}} \frac{H}{\sqrt{2 k^{3}}}\left\{H ^ { 2 } \left[\frac{\ln ^{3}(a)}{9}-\frac{\ln ^{2}(a)}{4}+\left(\frac{\pi^{2}}{18}+\frac{5}{48}\right) \ln (a)+\frac{\pi^{2}}{54}-\frac{1}{96}-\frac{2 \zeta(3)}{3}\right.\right. \\
& +\left(\frac{33}{16}-\frac{\pi^{2}}{6}\right) a^{-1}+\left(\frac{\pi^{2}}{12}-\frac{35}{96}\right) a^{-2}-\left(\frac{\pi^{2}}{54}+\frac{139}{1296}\right) a^{-3}+\frac{1}{3} \sum_{n=3}^{\infty} \frac{(13 n+1) a^{-(n+1)}}{(n-2)(n-1) n(n+1)^{3}} \\
& \left.+\sum_{p=2}^{\infty} \sum_{n=1}^{p-1} \frac{1}{n(p-n)}\left\{\frac{2}{p(p+1)(p+2)(p+3)}-a^{-p}\left(\frac{1}{3 p}-\frac{a^{-1}}{p+1}+\frac{a^{-2}}{p+2}-\frac{a^{-3}}{3(p+3)}\right)\right\}\right] \\
& +\frac{k^{2}}{a^{2}}\left[\left(\frac{13283}{54000}-\frac{\pi^{2}}{90}\right) a^{2}+\left(\frac{\pi^{2}}{18}-\frac{629}{432}\right) a+\frac{\ln ^{3}(a)}{18}+\frac{5 \ln ^{2}(a)}{24}+\left(\frac{\pi^{2}}{36}+\frac{37}{96}\right) \ln (a)\right. \\
& +\frac{53 \pi^{2}}{540}+\frac{2623}{43200}-\frac{\zeta(3)}{3}+\left(\frac{\pi^{2}}{36}+\frac{19}{480}\right) a^{-1}-\left(\frac{\pi^{2}}{72}+\frac{169}{2880}\right) a^{-2}+\left(\frac{\pi^{2}}{540}-\frac{353}{12960}\right) a^{-3} \\
& +\frac{863 a^{-4}}{21600}-\frac{2491 a^{-5}}{378000}+\frac{1}{30} \sum_{n=5}^{\infty} \frac{\left(65 n^{3}-190 n^{2}-193 n-58\right) a^{-(n+1)}}{(n-2)(n-1) n(n+1)^{3}(n+2)(n+3)}
\end{aligned}
$$




$$
\begin{aligned}
& +\sum_{p=3}^{\infty} \sum_{n=1}^{p-1} \frac{1}{n(p-n)}\left\{\frac{p^{2}+5 p+2}{(p-2)(p-1) p(p+1)(p+2)(p+3)}-a^{-(p-2)}\left(\frac{2}{15(p-2)}-\frac{a^{-1}}{3(p-1)}\right.\right. \\
& \left.\left.\left.+\frac{a^{-2}}{6 p}+\frac{a^{-3}}{6(p+1)}-\frac{a^{-4}}{6(p+2)}+\frac{a^{-5}}{30(p+3)}\right)\right\}\right]+\frac{i k^{3}}{H a^{3}}\left[\left(\frac{155}{1296}-\frac{\pi^{2}}{162}\right) a^{3}+\left(\frac{\pi^{2}}{36}-\frac{15}{32}\right) a^{2}\right. \\
& +\left(\frac{53}{48}-\frac{\pi^{2}}{18}\right) a-\frac{\ln ^{3}(a)}{27}-\frac{13 \ln ^{2}(a)}{108}-\left(\frac{\pi^{2}}{54}+\frac{329}{1296}\right) \ln (a)+\frac{845}{7776}-\frac{2 \pi^{2}}{27}+\frac{2 \zeta(3)}{9} \\
& +\frac{a^{-1}}{216}-\frac{233 a^{-2}}{2160}+\frac{41 a^{-3}}{1080}+\frac{1}{9} \sum_{n=3}^{\infty} \frac{(13 n+1) a^{-(n+1)}}{(n+1)^{3}(n+2)(n+3)(n+4)} \\
& \left.+\sum_{p=4}^{\infty} \sum_{n=1}^{p-1} \frac{1}{n(p-n)}\left\{\frac{2}{3(p-3)(p-2)(p-1) p}-a^{-(p-3)}\left(\frac{1}{9(p-3)}-\frac{a^{-1}}{3(p-2)}+\frac{a^{-2}}{3(p-1)}-\frac{a^{-3}}{9 p}\right)\right\}\right] \\
& +\frac{k^{4}}{H^{2} a^{4}}\left[\left(\frac{\pi^{2}}{420}-\frac{548167}{12348000}\right) a^{4}+\left(\frac{9403}{54000}-\frac{\pi^{2}}{90}\right) a^{3}+\left(\frac{\pi^{2}}{45}-\frac{8213}{27000}\right) a^{2}+\left(\frac{425}{864}-\frac{\pi^{2}}{36}\right) a\right. \\
& -\frac{\ln ^{3}(a)}{72}-\frac{5 n^{2}(a)}{96}-\left(\frac{\pi^{2}}{144}+\frac{127}{1152}\right) \ln (a)+\frac{37733}{564480}-\frac{467 \pi^{2}}{15120}+\frac{\zeta(3)}{12}-\left(\frac{857}{13440}+\frac{\pi^{2}}{720}\right) a^{-1} \\
& +\left(\frac{\pi^{2}}{1440}+\frac{24167}{403200}\right) a^{-2}-\left(\frac{\pi^{2}}{15120}+\frac{65183}{1814400}\right) a^{-3}+\frac{11 a^{-4}}{1152}+\frac{a^{-5}}{10080}-\frac{13 a^{-6}}{12096}+\frac{11 a^{-7}}{70560} \\
& -\frac{1}{840} \sum_{n=3}^{\infty} \frac{\left(455 n^{5}-875 n^{4}+3787 n^{3}-11109 n^{2}-8538 n-2392\right) a^{-(n+1)}}{(n-2)(n-1) n(n+1)^{3}(n+2)(n+3)(n+4)(n+5)} \\
& \left.\left.\left.-\sum_{p=5}^{\infty} \sum_{n=1}^{p-1} \frac{a^{-1}}{5(p-3)}+\frac{4 a^{-2}}{15(p-2)}-\frac{a^{-3}}{6(p-1)}+\frac{a^{-4}}{24 p}+\frac{a^{-5}}{120(p+1)}-\frac{a^{-6}}{120(p+2)}+\frac{a^{-7}}{840(p+3)}\right)\right\}+\mathcal{O}\left(\frac{i k^{5}}{H^{3}}\right)\right\} .
\end{aligned}
$$

Remaining integral $\mathcal{I}_{6}(\eta, k)$ involves trivial delta function integrations which yield

$$
\begin{aligned}
& \mathcal{I}_{6}(\eta, k)=\frac{-1}{2^{7} \pi^{4}}\left\{\left[\ln (a) \frac{\left(\partial_{0}^{2}+k^{2}\right)}{12 a^{2}}+(2 \ln (a)+1) H \frac{\partial_{0}}{12 a}\right]-H^{2}\left[\frac{4 \ln ^{3}(a)}{9}+\frac{23 \ln ^{2}(a)}{18}\right.\right. \\
& \left.\left.+\left(\frac{2 \pi^{2}}{9}+3 \ln \left(\frac{2 \mu}{H}\right)-\frac{13}{3}\right) \ln (a)+\frac{3 a^{-2}}{4}+\frac{8 a^{-3}}{81}+\sum_{n=4}^{\infty} \frac{(n-3)(n+2) a^{-(n+1)}}{(n-2)(n+1)^{3}}\right]\right\} u(\eta, k) .
\end{aligned}
$$

Acting the derivatives upon the tree-order mode function $u(\eta, k)$, we find

$$
\begin{aligned}
& \mathcal{I}_{6}(\eta, k)=\frac{1}{2^{7} \pi^{4}} \frac{H}{\sqrt{2 k^{3}}}\left\{H ^ { 2 } \left[\frac{4 \ln ^{3}(a)}{9}+\frac{23 \ln ^{2}(a)}{18}+\left(\frac{2 \pi^{2}}{9}+3 \ln \left(\frac{2 \mu}{H}\right)-\frac{13}{3}\right) \ln (a)+\frac{3 a^{-2}}{4}\right.\right. \\
& \left.+\frac{8 a^{-3}}{81}+\sum_{n=4}^{\infty} \frac{(n-3)(n+2) a^{-(n+1)}}{(n-2)(n+1)^{3}}\right]+\frac{k^{2}}{a^{2}}\left[\frac{2 \ln ^{3}(a)}{9}+\frac{23 \ln ^{2}(a)}{36}+\left(\frac{\pi^{2}}{9}+\frac{3}{2} \ln \left(\frac{2 \mu}{H}\right)-\frac{13}{6}\right) \ln (a)\right. \\
& \left.+\frac{1}{12}+\frac{3 a^{-2}}{8}+\frac{4 a^{-3}}{81}+\frac{1}{2} \sum_{n=4}^{\infty} \frac{(n-3)(n+2) a^{-(n+1)}}{(n-2)(n+1)^{3}}\right]+\frac{i k^{3}}{H a^{3}}\left[\frac{4 \ln ^{3}(a)}{27}+\frac{23 \ln ^{2}(a)}{54}\right.
\end{aligned}
$$




$$
\begin{array}{r}
\left.+\left(\frac{2 \pi^{2}}{27}+\ln \left(\frac{2 \mu}{H}\right)-\frac{13}{9}\right) \ln (a)+\frac{1}{12}+\frac{a^{-2}}{4}+\frac{8 a^{-3}}{243}+\frac{1}{3} \sum_{n=4}^{\infty} \frac{(n-3)(n+2) a^{-(n+1)}}{(n-2)(n+1)^{3}}\right] \\
-\frac{k^{4}}{H^{2} a^{4}}\left[\frac{\ln ^{3}(a)}{18}+\frac{23 \ln ^{2}(a)}{144}+\left(\frac{\pi^{2}}{36}+\frac{3}{8} \ln \left(\frac{2 \mu}{H}\right)-\frac{13}{24}\right) \ln (a)+\frac{1}{24}+\frac{3 a^{-2}}{32}+\frac{a^{-3}}{81}\right. \\
\left.\left.+\frac{1}{8} \sum_{n=4}^{\infty} \frac{(n-3)(n+2) a^{-(n+1)}}{(n-2)(n+1)^{3}}\right]+\mathcal{O}\left(\frac{i k^{5}}{H^{3}}\right)\right\} .
\end{array}
$$

In the next appendix, we sum up $\mathcal{I}_{1}(\eta, k)$ given in Eq. (86) and the five integrals we evaluated in this appendix to calculate source term (70) of integro-differential equation (69).

\section{Appendix C: Calculating the source term $\mathcal{S}(\eta, k)$}

In Eq. (70) we defined the non-homogeneous part of the integro-differential equation for the two-loop correction $\Phi_{2}(\eta, k)$ to the scalar mode function, as the source term

$$
\mathcal{S}(\eta, k) \equiv-\frac{H^{2}}{8 \pi^{2}} \ln (a(\eta)) \Phi_{1}(\eta, k)+\sum_{n=1}^{6} \mathcal{I}_{n}(\eta, k)
$$

The one-loop correction $\Phi_{1}(\eta, k)$ is given in Eqs. (61) and (62). The integrals $\left\{\mathcal{I}_{n}(\eta, k): n=\right.$ $1,2, \ldots, 6\}$ are given in Eqs. (86), (B3), (B12), (B17), (B21), (B23), respectively. Inserting these results into Eq. (C1) yields

$$
\begin{aligned}
& \mathcal{S}(\eta, k)=\frac{1}{2^{6} \pi^{4}} \frac{H}{\sqrt{2 k^{3}}}\left\{H ^ { 2 } \left[\frac{11 \ln ^{3}(a)}{18}+\frac{19 \ln ^{2}(a)}{36}+\left(\frac{2 \pi^{2}}{9}-\frac{559}{216}+\frac{3 \ln \left(\frac{2 \mu}{H}\right)}{2}\right) \ln (a)\right.\right. \\
& +\frac{\ln ^{2}\left(\frac{2 \mu}{H}\right)}{2}-\frac{5 \ln \left(\frac{2 \mu}{H}\right)}{6}+\frac{5}{12}+\frac{13 \pi^{2}}{108}-\frac{4 \zeta(3)}{3}+\frac{7 a^{-2}}{12}-\left(\frac{\ln (a)}{27}+\frac{\pi^{2}}{27}-\frac{109}{648}\right) a^{-3} \\
& +\sum_{n=3}^{\infty} \frac{\left(n^{7}-2 n^{6}-8 n^{5}+46 n^{4}+19 n^{3}-188 n^{2}+196 n+64\right) a^{-(n+1)}}{24(n-2)(n-1) n(n+1)^{3}}+2 \sum_{p=2}^{\infty} \sum_{n=1} \frac{1}{n(p-n)} \\
& \left.\times\left\{\frac{1}{p(p+3)}-\frac{a^{-p}}{4}\left[1-p\left(1-a^{-1}\right)+\frac{4}{3}\left(\frac{1}{p}-\frac{a^{-3}}{p+3}\right)\right]\right\}\right]+k^{2}\left[\frac{\ln (a)}{30}+\frac{13283}{27000}-\frac{\pi^{2}}{45}+a^{-2}\left[\frac{11 \ln ^{3}(a)}{36}\right.\right. \\
& \left.-\frac{29 \ln (a)}{72}+\left(\frac{\pi^{2}}{9}-\frac{151}{432}+\frac{3 \ln \left(\frac{2 \mu}{H}\right)}{4}\right) \ln (a)+\frac{\ln ^{2}\left(\frac{2 \mu}{H}\right)}{4}-\frac{5 \ln \left(\frac{2 \mu}{H}\right)}{12}-\frac{347}{1350}+\frac{77 \pi^{2}}{1080}-\frac{2 \zeta(3)}{3}\right] \\
& -\left(\frac{\ln (a)}{3}+\frac{\pi^{2}}{9}-\frac{59}{40}\right) a^{-3}-\frac{301 a^{-4}}{360}+\left(\frac{\ln (a)}{270}+\frac{\pi^{2}}{270}+\frac{5207}{6480}\right) a^{-5}+\frac{2671 a^{-6}}{21600}+\frac{909 a^{-7}}{7000} \\
& +\sum_{n=5}^{\infty} \frac{\left(5 n^{9}+35 n^{8}+40 n^{7}+10 n^{6}+645 n^{5}-905 n^{4}-6170 n^{3}-1860 n^{2}+6152 n+512\right) a^{-(n+3)}}{240(n-2)(n-1) n(n+1)^{3}(n+2)(n+3)} \\
& -\sum_{p=3}^{\infty} \sum_{n=1}^{p-1} \frac{1}{n(p-n)}\left\{\frac{2 a^{-2}}{(p-2) p(p+1)(p+3)}-a^{-p}\left[\frac{\left(5 p^{2}-5 p-8\right)}{30(p-2)}-\frac{\left(3 p^{2}+9 p+4\right) a^{-2}}{12 p}\right.\right.
\end{aligned}
$$




$$
\begin{aligned}
& \left.\left.\left.\left.+\frac{\left(p^{2}+5 p+8\right) a^{-3}}{12(p+1)}-\frac{a^{-5}}{15(p+3)}\right)\right]\right\}\right]+\frac{i k^{3}}{H}\left[\frac{\ln (a)}{81}+\frac{155}{648}-\frac{\pi^{2}}{81}+\frac{5 a^{-2}}{4}-\frac{\ln ^{3}(a)}{18 a^{3}}-\frac{7 \ln ^{2}(a)}{108 a^{3}}\right. \\
& +\left(\frac{2285}{4536}+\frac{\ln \left(\frac{2 \mu}{H}\right)}{2}\right) \frac{\ln (a)}{a^{3}}+\left(\frac{\ln ^{2}\left(\frac{2 \mu}{H}\right)}{6}-\frac{5 \ln \left(\frac{2 \mu}{H}\right)}{18}+\frac{4 \zeta(3)}{9}-\frac{13 \pi^{2}}{108}-\frac{12023}{13608}\right) a^{-3}-\frac{185}{108 a^{4}}+\frac{103}{270 a^{5}} \\
& +\frac{989}{4860 a^{6}}+\sum_{n=3}^{\infty} \frac{\left(n^{8}+14 n^{7}+68 n^{6}+146 n^{5}+239 n^{4}+80 n^{3}-2116 n^{2}-5056 n-2720\right) a^{-(n+4)}}{72(n-2)(n+1)^{3}(n+2)(n+3)(n+4)} \\
& \left.-\sum_{p=4 n=1}^{\infty} \sum_{n-1} \frac{1}{n(p-n)}\left\{\frac{(p+4) a^{-3}}{3(p-3)(p-2) p}-\frac{a^{-p}}{18}\left(\frac{3 p^{2}-3 p-4}{p-3}-\frac{3 p(p+1) a^{-1}}{p-2}+\frac{4 a^{-3}}{p}\right)\right\}\right] \\
& -\frac{k^{4}}{H^{2}}\left[\frac{\ln (a)}{280}-\frac{\pi^{2}}{210}+\frac{548167}{6174000}-\frac{\ln (a)}{60 a^{2}}+\left(\frac{\pi^{2}}{90}+\frac{4477}{54000}\right) a^{-2}-\frac{a^{-3}}{6}+\frac{11 \ln ^{3}(a)}{144 a^{4}}+\frac{19 \ln ^{2}(a)}{288 a^{4}}\right. \\
& -\left(\frac{199}{1728}-\frac{\pi^{2}}{36}-\frac{3 \ln \left(\frac{2 \mu}{H}\right)}{16}\right) \frac{\ln (a)}{a^{4}}+\left(\frac{88663}{235200}+\frac{1343 \pi^{2}}{30240}+\frac{\ln ^{2}\left(\frac{2 \mu}{H}\right)}{16}-\frac{5 \ln \left(\frac{2 \mu}{H}\right)}{48}-\frac{\zeta(3)}{6}\right) a^{-4} \\
& -\frac{\ln (a)}{30 a^{5}}-\left(\frac{\pi^{2}}{90}+\frac{719}{560}\right) a^{-5}+\frac{51577}{50400 a^{6}}+\frac{\ln (a)}{7560 a^{7}}-\left(\frac{113849}{907200}-\frac{\pi^{2}}{7560}\right) a^{-7}-\frac{7 a^{-8}}{40}+\frac{1297 a^{-9}}{25200} \\
& -\frac{a^{-10}}{2520}-\frac{11 a^{-11}}{35280}+\sum_{n=3}^{\infty} \frac{\left(455 n^{5}-875 n^{4}+3787 n^{3}-11109 n^{2}-8538 n-2392\right) a^{-(n+5)}}{420(n-2)(n-1) n(n+1)^{3}(n+2)(n+3)(n+4)(n+5)} \\
& +\sum_{n=3}^{\infty} \frac{\left(5 n^{8}+80 n^{7}+450 n^{6}+1160 n^{5}+1805 n^{4}+760 n^{3}+252 n^{2}-5664 n-1856\right) a^{-(n+5)}}{960(n-1) n(n+1)^{2}(n+2)(n+3)(n+4)} \\
& +\sum_{n=3}^{\infty} \frac{(n-3)(n+2) a^{-(n+5)}}{16(n-2)(n+1)^{3}}-\sum_{p=5}^{\infty} \sum_{n=1}^{p-1} \frac{1}{n(p-n)}\left\{\frac{\left(p^{4}+8 p^{3}+21 p^{2}+22 p+24\right) a^{-4}}{4(p-4)(p-3)(p-2) p(p+1)(p+3)}\right. \\
& -a^{-p}\left[\frac{7 p^{2}-7 p-8}{70(p-4)}-\frac{p(p+1) a^{-1}}{6(p-3)}+\frac{\left(5 p^{2}+15 p+8\right) a^{-2}}{60(p-2)}-\frac{\left(p^{2}+7 p+4\right) a^{-4}}{48 p}\right. \\
& +\left(p^{2}+9 p+16\right) a^{-5} \\
& \left.\left.\left.\left.\frac{a^{-7}}{420(p+3)}\right]\right\}\right]+\mathcal{O}\left(\frac{i k^{5}}{H^{3}}\right)\right\}
\end{aligned} .
$$

When the source term $(\mathrm{C} 2)$ is integrated against the Green's function (57) in Eq. (87) gives the two-loop correction $\Phi_{2}(\eta, k)$ of Eq. (89).

\section{Appendix D: Evaluating the functional $\mathcal{W}(\alpha)$}

In calculating the third integral $I_{3}(\eta, k)$ of the source term $\mathcal{S}(\eta, k)$ in Appendix B we defined the functional $\mathcal{W}(\alpha)$ in Eq. (B8) as

$$
\begin{aligned}
\mathcal{W}(\alpha) & =\int_{0}^{1} d z z \sin (\alpha z) \ln ^{2}\left(\frac{1-z^{2}}{4}\right) \\
& =\sum_{n=0}^{\infty} \frac{(-1)^{n} \alpha^{2 n+1}}{(2 n+1) !} \int_{0}^{1} d z z^{2 n+2} \ln ^{2}\left(\frac{1-z^{2}}{4}\right) .
\end{aligned}
$$


In this appendix we calculate the $\mathcal{W}(\alpha)$. To evaluate the integral in Eq. (D2), let us first consider the following integral

$$
\int_{0}^{1} d z z^{2 n+2}\left(\frac{1-z^{2}}{4}\right)^{b}
$$

Making the change of variable $z^{2} \equiv t$ yields

$$
\begin{aligned}
\int_{0}^{1} d z z^{2 n+2}\left(\frac{1-z^{2}}{4}\right)^{b} & =\frac{1}{2^{2 b+1}} \int_{0}^{1} d t t^{n+\frac{1}{2}}(1-t)^{b}=\frac{1}{2^{2 b+1}} \mathcal{B}\left(n+\frac{3}{2}, b+1\right) \\
& =\frac{\Gamma(1+b) \Gamma\left(\frac{3}{2}+n\right)}{2^{2 b+1} \Gamma\left(\frac{5}{2}+b+n\right)}
\end{aligned}
$$

which is valid for arbitrary $b$ and $n$ as long as $\operatorname{Re}[b]>-1$ and $\operatorname{Re}[n]>-\frac{3}{2}$. $\mathcal{B}$ denotes the Beta function. Differentiating the both sides of Eq. (D4) and taking $b \rightarrow 0$, we obtain the desired integral in Eq. (D2) as

$$
\int_{0}^{1} d z z^{2 n+2} \ln ^{2}\left(\frac{1-z^{2}}{4}\right)=\frac{\Gamma\left(\frac{3}{2}+n\right)}{2 \Gamma\left(\frac{5}{2}+n\right)}\left\{4 \ln (2)\left[\ln (2)+\mathcal{H}_{\frac{3}{2}+n}\right]-\psi^{\prime}\left(\frac{5}{2}+n\right)+\mathcal{H}_{\frac{3}{2}+n}^{2}+\frac{\pi^{2}}{6}\right\}
$$

where the Digamma Function $\psi(z)$ is the logarithmic derivative of the Gamma Function, $\psi(z)=\frac{\Gamma^{\prime}(z)}{\Gamma(z)}$, and the Harmonic Number Function $\mathcal{H}_{\frac{3}{2}+n}=\gamma+\psi\left(\frac{5}{2}+n\right)$. Prime denotes the derivative with respect to the argument. Note that, if we had done the calculation of the third integral $I_{3}(\eta, k)$ in a way that the factor $1 / 4$ in the argument of the logarithm had not been included in definition $(\mathrm{B} 8)$ of the $\mathcal{W}(\alpha)$, the first term on the right hand side of Eq. (D5) would not have been present there. Although Eq. (D5) would look compact, that result, however, would yield complicated terms for each integer $n$. Equation (D5), on the other hand, yields simpler terms for $n=0, n=1$ and $n=2$,

$$
\begin{aligned}
\int_{0}^{1} d z z^{2} \ln ^{2}\left(\frac{1-z^{2}}{4}\right) & =\frac{104}{27}-\frac{\pi^{2}}{9} \\
\int_{0}^{1} d z z^{4} \ln ^{2}\left(\frac{1-z^{2}}{4}\right) & =\frac{3152}{1125}-\frac{\pi^{2}}{15}, \\
\int_{0}^{1} d z z^{6} \ln ^{2}\left(\frac{1-z^{2}}{4}\right) & =\frac{175568}{77175}-\frac{\pi^{2}}{21},
\end{aligned}
$$

respectively. (If we had odd powers of $z$ in Eq. (D5), then the result of the integral for each integer $n$ would be simpler without the inclusion of the factor $1 / 4$ in the argument of the logarithm, in the definition of the $\mathcal{W}(\alpha)$.) Using Eqs. (D6)-(D8) in Eq. (D2) we obtain

$$
\mathcal{W}(\alpha)=\left(\frac{104}{27}-\frac{\pi^{2}}{9}\right) \alpha-\left(\frac{1576}{3375}-\frac{\pi^{2}}{90}\right) \alpha^{3}+\left(\frac{21946}{1157625}-\frac{\pi^{2}}{2520}\right) \alpha^{5}+\mathcal{O}\left(\alpha^{7}\right) .
$$

This functional also appears in the evaluations of the integrals $I_{4}(\eta, k)$ and $I_{5}(\eta, k)$. See Eqs. (B15) and (B19), respectively. 


\section{Acknowledgments}

I am grateful to Richard P. Woodard for stimulating discussions.

[1] R. P. Woodard, "Quantum Effects during Inflation," in Quantum Field Theory under the Influence of External Conditions (Rinton Press, Princeton, 2004) ed. K. A. Milton, pp. 325330, astro-ph/0310757.

[2] V. F. Mukhanov and G. V. Chibisov, JETP Lett. 33 (1981) 532.

[3] A. A. Starobinskiı̌, JETP Lett. 30 (1979) 682.

[4] V. K. Onemli and R. P. Woodard, Class. Quant. Grav. 19 (2002) 4607.

[5] V. K. Onemli and R. P. Woodard, Phys. Rev. D70 (2004) 107301.

[6] E. O Kahya, V. K. Onemli and R. P. Woodard, Phys. Rev. D81 (2010) 023508.

[7] T. Brunier, V. K. Onemli and R. P. Woodard, Class. Quant. Grav. 22 (2005) 59.

[8] E. O. Kahya and V. K. Onemli, Phys. Rev. D76 (2007) 043512.

[9] T. Prokopec and R. P. Woodard, JHEP 0310 (2003) 059.

[10] L. D. Duffy and R. P. Woodard, Phys. Rev. D72 (2005) 024023.

[11] S. P. Miao and R. P. Woodard, Phys. Rev. D74 (2006) 044019.

[12] T. Prokopec, O. Tornkvist and R. P. Woodard, Phys. Rev. Lett. 89 (2002) 101301; Ann. Phys. 303 (2003) 251; T. Prokopec and R. P. Woodard, Ann. Phys. 312 (2004) 1.

[13] E. O. Kahya and R. P. Woodard, Phys. Rev. D72 (2005) 104001; Phys. Rev. D74 (2006) 084012 .

[14] S. P. Miao and R. P. Woodard, Class. Quant. Grav. 23 (2006) 1721; Phys. Rev. D74 (2006) 024021.

[15] E. O. Kahya and R. P. Woodard, Phys. Rev. D77 (2008) 084012.

[16] J. Schwinger, J. Math. Phys. 2 (1961) 407; K. T. Mahanthappa, Phys. Rev. 126 (1962) 329; P. M. Bakshi and K. T. Mahanthappa, J. Math. Phys. 4 (1963) 1; L. V. Keldysh, Sov. Phys. JETP 20 (1965) 1018.

[17] R. D. Jordan, Phys. Rev. D33 (1986) 444; K. C. Chou, Z. B. Su, B. L. Hao and L. Yu, Phys. Rept. 118 (1985) 1; E. Calzetta and B. L. Hu, Phys. Rev. D35 (1987) 495.

[18] S. P. Miao and S. Park, arXiv:1306.4126. 
[19] J. Maldacena, JHEP 0305 (2003) 013; S. Weinberg, Phys. Rev. D72 (2005) 043514; Phys. Rev. D74 (2006) 023508; D. Boyanovsky, H. J. de Vega and N. G. Sanchez, Nucl. Phys. B747 (2006) 25; Phys. Rev. D72 (2005) 103006; M. Sloth, Nucl. Phys. B748 (2006) 149; Nucl. Phys. B775 (2007) 78; D. Seery, J. E. Lidsey and M. S. Sloth, JCAP 0701 (2007) 027; M. van der Meulen and J. Smit, JCAP 0711 (2007) 023; D. H. Lyth, JCAP 0712 (2007) 016; D. Seery, JCAP 0711 (2007) 025; JCAP 0802 (2008) 006; JCAP 0905 (2009) 021; Class. Quant. Grav. 27 (2010) 124005; N. Bartolo, S. Matarrese, M. Pietroni, A. Riotto and D. Seery, JCAP 0801 (2008) 015; Y. Urakawa and K. I. Maeda, Phys. Rev. D78 (2008) 064004; A. Riotto and M. Sloth, JCAP 0804 (2008) 030; JCAP 1110 (2011) 003; K. Enqvist, S. Nurmi, D. Podolsky and G. I. Rigopoulos, JCAP 0804 (2008) 025; P. Adshead, R. Easther and E. A. Lim, Phys. Rev. D79 (2009) 063504; E. O. Kahya, V. K. Onemli and R. P. Woodard, Phys. Lett. B694 (2010) 101; Y. Urakawa and T. Tanaka, Prog. Theor. Phys. 122 (2009) 779; Prog. Theor. Phys. 122 (2010) 1207; Phys. Rev. D82 (2010) 121301; Prog. Theor. Phys. 125 (2011) 1067; JCAP 1105 (2011) 014; Prog. Theor. Exp. Phys. 2013 (2013) 6, 063E02; Prog. Theor. Exp. Phys. 2013 (2013) 8 083E01; Y. Urakawa, Prog. Theor. Phys. 126 (2011) 961; S. B. Giddings and M. S. Sloth, JCAP 1007 (2010) 015; JCAP 1101 (2011) 023; Phys. Rev. D84 (2011) 063528; Phys. Rev. D86 (2012) 083538; J. Kumar, L. Leblond and A. Rajaraman, JCAP 1004 (2010) 024; C. P. Burgess, R. Holman, L. Leblond and S. Shandera, JCAP 1003 (2010) 033; L. Senatore and M. Zaldarriaga, JHEP 1012 (2010) 008; M. Gerstenlauer, A. Hebecker and G. Tasinato, JCAP 1106 (2011) 021; W. Xue, X. Gao and R. Brandenberger, JCAP 1206 (2012) 035; G. L. Pimental, L. Senatore and M. Zaldarriaga, JHEP 1207 (2012) 166; S. P. Miao and R. P. Woodard, JCAP 1207 (2012) 008; M. G. Romania, N. C. Tsamis and R. P. Woodard, JCAP 1208 (2012) 029; D. Boyanovsky, Phys. Rev. D 85 (2012) 123525; Phys. Rev. D 86 (2012) 023509; K. Feng, Y.-F. Cai and Y.-S. Piao, Phys. Rev. D 86 (2012) 103515; A. Kaya, Phys. Rev. D 86 (2012) 123511; arXiv:1306.3236; R. Brandenberger and J. Martin, Class. Quant. Grav. 30 (2013) 113001; Y. Korai and T. Tanaka, Phys. Rev. D 87 (2013) 024013; E.T. Akhmedov, Int. J. Mod. Phys. D23 (2014) 1430001; K. Larjo and D. A. Lowe, Phys. Rev. D 87 (2013) 083506; J. Serreau and R. Parentani, Phys. Rev. D 87 (2013) 085012; J. Serreau, arXiv:1302.6365; L. Lello, D. Boyanovsky and R. Holman arXiv:1307.4066.

[20] F. L. Bezrukov and M. E. Shaposhnikov, Phys. Lett. B659 (2008) 703. 